This item was submitted to Loughborough's Research Repository by the author.

Items in Figshare are protected by copyright, with all rights reserved, unless otherwise indicated.

\title{
A theory of one-dimensional fracture
}

PLEASE CITE THE PUBLISHED VERSION

http://dx.doi.org/10.1016/j.compstruct.2011.09.011

PUBLISHER

(c) Elsevier

VERSION

AM (Accepted Manuscript)

LICENCE

CC BY-NC-ND 4.0

REPOSITORY RECORD

Wang, Simon, and Christopher M. Harvey. 2011. "A Theory of One-dimensional Fracture". figshare. https://hdl.handle.net/2134/9071. 
This item was submitted to Loughborough's Institutional Repository (https://dspace.lboro.ac.uk/) by the author and is made available under the following Creative Commons Licence conditions.

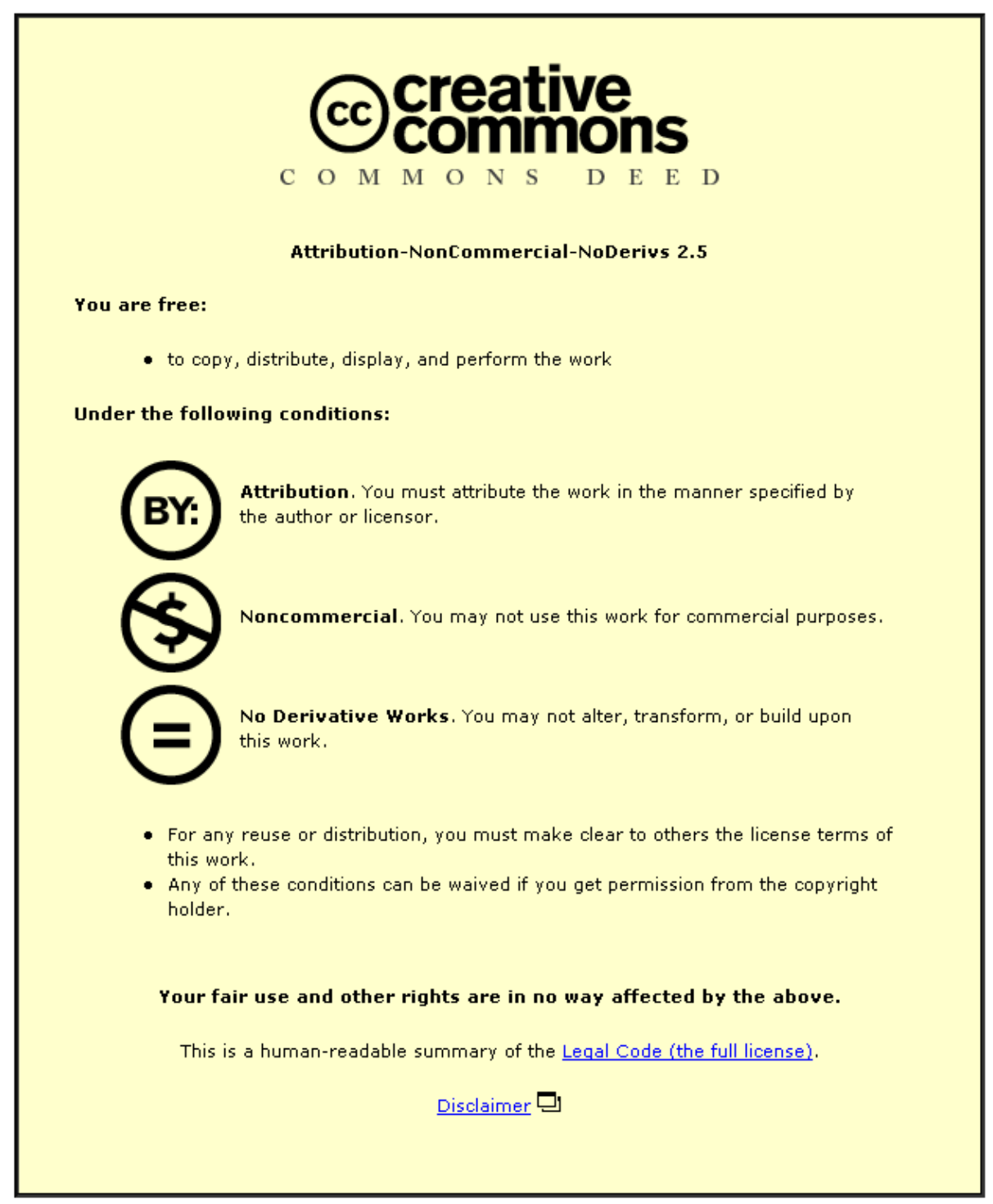

For the full text of this licence, please go to: http://creativecommons.org/licenses/by-nc-nd/2.5/ 


\title{
A theory of one-dimensional fracture
}

\author{
S. Wang ${ }^{*}$ and C. Harvey \\ Department of Aeronautical and Automotive Engineering, Loughborough University, Loughborough, \\ Leicestershire LE11 3TU, UK
}

\begin{abstract}
A completely analytical theory is developed for the mixed mode partition of one-dimensional fracture in laminated composite beams and plates. Two sets of orthogonal pure modes are determined first. It is found that they are distinct from each other in Euler beam or plate theory and coincide at the Wang-Harvey set in Timoshenko beam or plate theory. After the WangHarvey set is proved to form a unique complete orthogonal pure mode basis within the contexts of both Euler and Timoshenko beam or plate theories, it is used to partition a mixed mode. Stealthy interactions are found between the Wang-Harvey pure mode I modes and mode II modes in Euler beam or plate theory, which alter the partitions of a mixed mode. The finite element method is developed to validate the analytical theories.
\end{abstract}

Keywords: Composite beams and plates, Energy release rate, Fracture, Mixed mode partition, Orthogonal pure modes

\section{Introduction}

Some typical examples of one-dimensional cracks in isotropic materials or one-dimensional delamination in laminated composite materials are shown in Fig. 1. For the sake of convenience, one-dimensional cracks and delamination are both called one-dimensional fracture in this work. Although fracture of engineering materials or structures is in general a three-dimensional mechanical problem, study of one-dimensional fracture still has great importance due to several reasons. One-dimensional fracture is often used in experimental tests, such as double cantilever beam (DCB) and end-notched flexure (ENF) tests, to obtain critical energy release rate or toughness of a material in either pure mode I or mode II fracture. In the case of a mixed mode, it is often used to investigate fracture propagation criteria. Moreover, many practical fractures in materials can be approximated as one-dimensional fracture. The most common ones are throughwidth fracture in straight or curved beams as shown in Fig. 1, circular ring-type fracture in plates and shells in a drilling process (also shown), separation of two material layers in a bio-cell under a needle puncture, separation of stiffeners and skins in stiffened plate or shell panels, etc.

The study in this paper focuses on brittle one-dimensional fracture. That is, the materials in consideration are assumed to be linearly elastic. The primary goal is to develop theories to find pure fracture modes and partition a mixed mode into pure modes. By achieving this goal, the aim is to provide academic researchers with theoretical tools for determining critical energy release rates of pure modes and developing fracture propagation criterion, and industrial engineers with design guidelines.

* Corresponding Author

Email addresses: s . wang@lbor . ac .uk (S. Wang), c . m . harvey@lbor o . ac . uk (C. Harvey) 
An important pioneering work on the topic [1] was given by Williams for isotropic DCBs based on Euler beam theory. A pair of pure modes I and II were correctly given. However, the partition of a mixed mode was in error. Another piece of pioneering work [2] was given by Schapery and Davidson, which was also for DCBs based on Euler beam theory. The work [2] was not able to give the Williams pair of pure modes [1] and claimed that Euler beam theory did not provide quite enough information to obtain a decomposition of energy release rate into opening and shearing mode components. Hutchinson and Suo [3] and Suo and Hutchinson [4] reported their work on isotropic DCBs based on a combined Euler beam theory and 2D elasticity with the use of stress intensity factors. The work [3,4] gave a combined numerical and analytical approach and was also unable to reproduce the Williams pair of pure modes [1]. The work [3] claimed the work [1] contained conceptual errors. Some other earlier works are given in Refs. $[5,6]$. Several recent research works on the topic are quoted here among many others. These are Chen et al. [7], Chen [8], Zou et al. [9], Wang and Qiao [10], Li et al. [11], Nguyen and Levy [12], Yan and Shang [13] and Ouyang and Li [14].

Recently, the authors have developed completely analytical theories for one-dimensional fractures in straight beams and axisymmetric plates made of either isotropic or laminated composite materials. Both classical and first order shear deformable beam or plate theories are used. The work has been reported on several occasions [15-19]. In this paper the essential theories for isotropic DCBs [20] and laminated composite DCBs [21] are derived first and extensions are then made to beams and axisymmetric plates with central fractures. The developed theories are validated using the finite element method (FEM) with Timoshenko beam and fournode plane-stress quadrilateral (QUAD4) elements.

\begin{tabular}{|c|c|}
\hline \multicolumn{2}{|c|}{ NOMENCLATURE } \\
\hline$a$ & length of fracture \\
\hline$A_{1}, A_{2}, A$ & cross-sectional area or extensional stiffness of upper, lower and intact beams \\
\hline$b$ & beam width \\
\hline$B_{1}, B_{2}, B$ & coupling stiffness of upper, lower and intact beams \\
\hline$D_{1}, D_{2}, D$ & bending stiffness of upper, lower and intact beams \\
\hline$D_{g p}, D_{s h}$ & crack tip relative opening and shearing displacements \\
\hline$D_{o p P}$ & crack tip relative opening displacement due to through-thickness shearing \\
\hline$E$ & Young's modulus \\
\hline$F_{n B}, F_{n B}$ & crack tip normal and shear forces \\
\hline$F_{n B P}$ & crack tip normal force due to through-thickness shearing \\
\hline$G, G_{I}, G_{I I}$ & total, mode I and mode II energy release rates \\
\hline$G_{P}$ & mode I energy release rate due to through-thickness shearing \\
\hline$G_{1 z 2}$ & through-thickness shearing modulus \\
\hline$G_{\theta}, G_{\beta}$ & energy release rates due to $\theta$ mode and $\beta$ mode \\
\hline$h_{1}, h_{2}, h$ & thicknesses of upper, lower and intact beams \\
\hline$H_{1}, H_{2}, H$ & through-thickness shearing stiffness of upper, lower and intact beams \\
\hline$I_{1}, I_{2}, I$ & second moments of area of upper, lower and intact beams \\
\hline
\end{tabular}




\begin{tabular}{|ll|}
\hline$k^{2}$ & through-thickness shear correction factor \\
$L_{1}, L_{2}$ & lengths of left and right intact parts of beam \\
$M_{1 B}, M_{2 B}, M_{B}$ & bending moments on upper, lower and intact beams at crack tip \\
$N_{1 B}, N_{2 B}, N_{B}$ & axial forces on upper, lower and intact beams at crack tip \\
$P_{1}, P_{2}$ & shear loads acting on upper and lower beams \\
$P_{1 B}, P_{2 B}, P_{B}$ & shear forces on upper, lower and intact beams at crack tip \\
$u_{1}, u_{2}$ & axial displacements of the upper and lower beams \\
$w_{1}, w_{2}$ & deflections of upper and lower beams \\
$x_{p}$ & distance from left crack tip to loading location \\
$\alpha$ & mixed mode partition coefficients \\
$\beta$ & pure mode II \\
$\gamma$ & thickness ratio $h_{2} / h_{1}$ \\
$\Delta G$ & interaction between pure modes \\
$\theta$ & pure mode I \\
$\varphi_{\theta}, \varphi_{3}$ & modal vectors for pure modes I and II \\
Abbreviations & \\
CLT & classical lamination theory \\
DCB & double cantilever beam \\
ENF & find-notched flexure \\
FEM & four-node plane-stress quadrilateral element \\
QUAD4 &
\end{tabular}

\section{Fundamental theory}

\subsection{Isotropic double cantilever beams}

Fig. 2 shows a DCB with its associated geometry, two tip bending moments and two tip axial forces. By considering the strain energy $U$, the energy release rate $G$ at the crack tip point B is obtained as

$$
\begin{aligned}
G=\frac{1}{b} \frac{\partial U}{\partial a}= & \frac{1}{2 b E}\left[\frac{N_{1 B t}^{2} \gamma}{b h_{1}(1+\gamma)}+\frac{M_{1 B}^{2}}{I_{1}}+\frac{M_{2 B}^{2}}{I_{2}}-\frac{1}{I}\left(M_{1 B}+M_{2 B}-\frac{h_{2} N_{1 B e}}{2}\right)^{2}\right] \\
& =\frac{1}{2}\left\{\begin{array}{lll}
M_{1 B} & M_{2 B} & N_{1 B e}
\end{array}\right\}[C]\left\{\begin{array}{lll}
M_{1 B} & M_{2 B} & N_{1 B e}
\end{array}\right\}^{T}
\end{aligned}
$$

where $\gamma=h_{2} / h_{1}$ is the thickness ratio, $N_{1 B e}=N_{1 B}-N_{2 B} / \gamma$ is the effective axial force and $[C]$ is the coefficient matrix and positive definite. By using the virtual crack closure technique the energy release rate $G$ in a mixed mode can be decomposed into pure mode I and mode II energy release rates as $G=G_{I}+G_{I I}$ with 


$$
G_{I}=\frac{F_{n B B} D_{o p}}{2 b \delta a} \quad \text { and } \quad G_{H I}=\frac{F_{s B} D_{s h}}{2 b \delta a}
$$

where $F_{n B}$ and $F_{s B}$ are the respective crack tip normal and shear forces, and $D_{b p}$ and $D_{s h \text {, }}$ are the respective relative opening and shearing displacements between the cracked two interfaces just behind the crack tip. The $\delta a$ in Eq. (2) represents an infinitesimal distance between the crack tip B and the point where $D_{o p}$ and $D_{s h}$ are calculated. The pure mode I fracture is obtained when $G_{I I}=0$ requiring either $D_{s h}=0$ or $F_{s B}=0$ or $D_{s h}=F_{s B}=0$. Similarly, the pure mode II

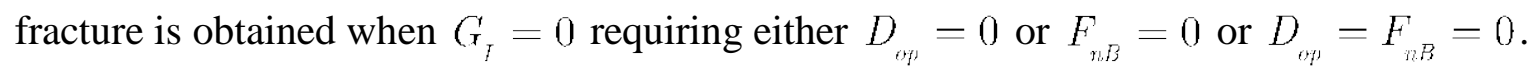

It is not a straightforward matter to calculate $F_{n B}$ and $F_{s B}$. However $D_{s h}$ and $D_{b p}$ can be easily obtained.

$$
\begin{gathered}
D_{s h}=\frac{1}{2 E}\left[\frac{h_{1} M_{1 B}}{I_{1}}+\frac{h_{2} M_{2 B}}{I_{2}}+\frac{2 N_{1 B e}}{A_{1}}\right] \delta a \\
D_{c p}=\frac{1}{2 E}\left(\frac{M_{1 B}}{I_{1}}-\frac{M_{2 B}}{I_{2}}\right) \delta a^{2}+\left(\psi_{1 B}-\psi_{2 B}\right) \delta a
\end{gathered}
$$

The cross-sectional rotations $\psi_{1 B}$ and $\psi_{2 B}$ in Eq. (4) are the crack tip rotations of beam 1 and 2, respectively. $D_{s h}=0$ yields

$$
6\left(\gamma^{2} M_{1 B}+M_{2 B}\right)+h_{1} \gamma^{2} N_{1 B e}=0
$$

From this condition two independent pure mode I modes are obtained.

$$
\varphi_{\theta_{1}}=\left\{\begin{array}{c}
1 \\
\theta_{1} \\
0
\end{array}\right\}=\left\{\begin{array}{c}
1 \\
-\gamma^{2} \\
0
\end{array}\right\} \text { and } \varphi_{\theta_{1}}=\left\{\begin{array}{c}
1 \\
0 \\
\theta_{2}
\end{array}\right\}=\left\{\begin{array}{c}
1 \\
0 \\
-6 / h_{1}
\end{array}\right\}
$$

Two independent pure mode II modes can be obtained by using orthogonal conditions.

$$
\varphi_{\beta_{1}}=\left\{\begin{array}{c}
1 \\
\beta_{1} \\
0
\end{array}\right\}=\left\{\begin{array}{c}
1 \\
\gamma^{2}(3+\gamma) /(1+3 \gamma) \\
0
\end{array}\right\} \text { and } \varphi_{\beta_{2}}=\left\{\begin{array}{c}
1 \\
0 \\
\beta_{2}
\end{array}\right\}=\left\{\begin{array}{c}
1 \\
0 \\
2(3+\gamma) /\left[h_{1}(\gamma-1)\right]
\end{array}\right\}
$$

When $h_{1}=h_{2}$

$$
\varphi_{\beta_{2}}=\left\{\begin{array}{c}
0 \\
0 \\
\beta_{2}
\end{array}\right\}=\left\{\begin{array}{l}
0 \\
0 \\
1
\end{array}\right\}
$$

Note that by 'orthogonal condition' it is meant that $\varphi_{\theta_{1}}^{T}[C] \varphi_{\beta_{1}}=0$ and $\varphi_{\theta_{2}}^{T I}[C] \varphi_{\beta_{2}}=0$. Moreover, it is also easy to show that $\varphi_{\theta_{1}}^{T}[C] \varphi_{\beta_{2}}=0$ and $\varphi_{\theta_{2}}^{T}[C] \varphi_{\beta_{1}}=0$ as pure mode II must be 
orthogonal to pure mode I. Moreover, both $\varphi_{3}$ and $\varphi_{\beta_{3}}$ are pure mode II modes corresponding to $F_{n, B}=0$ which is the orthogonal condition of $D_{s h_{n}}=0$. This set of pure mode pairs is called the Wang-Harvey pair. Details of this pair can be found in [20]. Therefore, a mixed mode can be decomposed into any three of the four pure modes given in Eqs. (6) and (7). In this work $\varphi_{0}$, $\varphi_{\beta_{1}}$ and $\varphi_{\beta_{2}}$ are used. That is,

$$
\left\{\begin{array}{lll}
M_{1 B} & M_{2 B} & N_{1 B C}
\end{array}\right\}^{T}=\alpha_{\beta_{1}} \varphi_{\theta_{1}}+\alpha_{\beta_{1}} \varphi_{\beta_{1}}+\alpha_{\beta_{2}} \varphi_{\beta_{2}}
$$

where $\alpha$ are mode partition coefficients and can be easily determined for a given left hand side of Eq. (8), i.e. a mixed mode. Substituting Eq. (8) into Eq. (1) gives

$$
G=\alpha_{\theta_{1}}^{2} G_{\theta_{1}}+\alpha_{\beta_{1}}^{2} G_{\beta_{1}}+\alpha_{\beta_{2}}^{2} G_{\beta_{2}}+\alpha_{\beta_{1}} \alpha_{\beta_{2}} G_{\beta_{1}}
$$

The first three terms in the right hand side of Eq. (9) come from the three pure modes $\varphi_{\theta_{1}}, \varphi_{\beta_{1}}$ and $\varphi_{\beta_{2}}$, respectively. The last term is from the coupling of $\varphi_{\beta_{1}}$ and $\varphi_{\beta_{2}}$ modes. Since the first term is from the pure mode I mode and the other three terms are from pure mode II modes, it may easily be concluded that

$$
G_{I}=\alpha_{\theta_{1}}^{2} G_{\theta_{1}} \text { and } G_{I I}=\alpha_{\beta_{1}}^{2} G_{\beta_{1}}+\alpha_{\beta_{2}}^{2} G_{\beta_{2}}+\alpha_{\beta_{1}} \alpha_{\beta_{2}} G_{\beta_{1} \beta_{2}}
$$

where

$$
G_{\theta_{1}}=\frac{24 \gamma}{E b^{2} h_{1}^{3}(1+\gamma)}
$$

However, extra care has to be taken. So far, there has been no difference in the development between Euler beam and Timoshenko beam theories. It will be shown now that the difference in two beam theories has a vital influence on mixed mode partition. A detailed rigorous treatment is given in [20].

A rigid un-cracked interface is assumed ahead of the crack tip B. Euler beam theory is considered first. Since the theory is of $C^{1}$ continuity, both deflection and rotation at the crack tip $\mathrm{B}$ are continuous and single valued. That is, $w_{1 B}=w_{2 B}=w_{B}$ and $\psi_{1 B}=\left(d w_{1} / d x\right)_{B}=\psi_{2 B}=\left(d w_{2} / d x\right)_{B}=\psi_{B}=(d w / d x)_{B}$. The $D_{o p}$ in Eq. (4) therefore becomes

$$
D_{v P}=\frac{1}{2 E}\left(\frac{M_{1 B}}{I_{1}}-\frac{M_{2 B}}{I_{2}}\right) \delta a^{2}
$$

in Euler beam theory. Letting $D_{o p}$ equal to zero gives another pure mode II.

$$
\varphi_{\beta_{E}^{\prime}}=\left\{\begin{array}{c}
1 \\
\beta_{F}^{\prime} \\
0
\end{array}\right\}=\left\{\begin{array}{c}
1 \\
\gamma^{3} \\
0
\end{array}\right\}
$$

Its orthogonal pure mode I is found to be 


$$
\varphi_{\theta_{E}^{\prime}}=\left\{\begin{array}{c}
1 \\
\theta_{F}^{\prime} \\
0
\end{array}\right\}=\left\{\begin{array}{c}
1 \\
-1 \\
0
\end{array}\right\}
$$

which corresponds to zero crack tip shear force, i.e. $F_{s B}=0$. Note that this pair of pure modes happens to be the Williams pair [1]. Now, it is seen that the crack tip shearing force $F_{s B}$ in pure mode I modes $\varphi_{\theta_{1}}$ and $\varphi_{\theta_{2}}$ is not zero whilst the relative crack opening displacement $D_{o p}$ in pure mode II modes $\varphi_{\beta_{1}}$ and $\varphi_{\beta_{2}}$ is not zero. Therefore, there exist interactions between the pure mode I modes, i.e. $\varphi_{\theta_{1}}$ and $\varphi_{\theta_{2}}$, and the pure mode II modes, i.e. $\varphi_{\beta_{1}}$ and $\varphi_{\beta_{2}}$. That is, both the crack tip normal force $F_{n B}$ and shearing force $F_{s B}$ in $\varphi_{\theta_{1}}$ and $\varphi_{\theta_{2}}$ will do work on the relative crack tip opening displacement $D_{o p}$ and shearing displacement $D_{s h}$ in $\varphi_{\beta_{1}}$ and $\varphi_{\beta_{2}}$ when fracture occurs. Since they are orthogonal to each other, the interactions result in zero net energy release rate. They are called stealthy interactions in this work because they alter the energy release rate partitions. In conclusion, Eq. (10) is not the correct partition in Euler beam theory and the stealthy interactions have to be considered. The crack tip opening force $F_{n B}$ in $\varphi_{0_{1}}$ can be calculated by using the first equation of Eq. (2) and Eqs. (11) and (12). Thus, the work done by $F_{n B}$ in $\varphi_{\theta_{1}}$ on $D_{o p}$ in $\varphi_{\beta_{1}}$ and $\varphi_{\beta_{2}}$ can be determined and the stealthy interactions are found to be

$$
\begin{gathered}
\Delta G_{\theta_{1} \beta_{1}}=\frac{3(\gamma-1)}{1+3 \gamma} G_{\theta_{1}} \\
\Delta G_{\theta_{1} \beta_{2}}=\frac{\gamma}{1+\gamma} G_{\theta_{1}}
\end{gathered}
$$

When $\gamma=1, \Delta G_{\theta_{1}, 3}=0$. Therefore, a mixed mode in Euler beam theory is partitioned as

$$
G_{I}=\alpha_{\theta_{1}}^{2} G_{\theta_{1}}+\alpha_{\theta_{1}} \alpha_{\beta_{1}} \Delta G_{\theta_{1} \beta_{1}}+\alpha_{\theta_{1}} \alpha_{\beta_{2}} \Delta G_{\theta_{1} \beta_{2}} \text { and } G_{I I}=G-G_{I}
$$

Next, Timoshenko beam theory is considered. Since the rotation becomes an independent variable of deformation instead of $\psi=d w / d x$, the two crack tip rotations $\psi_{1 B}$ and $\psi_{2 B}$ are generally different. Therefore, relative crack tip opening displacement $D_{o p}$ in Eq. (4) becomes

$$
D_{o p}=\left(\psi_{1 B}-\psi_{2 B}\right) \delta a
$$

Mechanically, the difference between $\psi_{1 B}$ and $\psi_{2 B}$ is due to the through-thickness shear effect. It is expected therefore that $\psi_{1 B}$ and $\psi_{2 B}$ will be equal to each other when crack tip normal force $F_{n B}$ disappears eliminating the through-thickness shear effect at the crack tip. A detailed study [20] confirms it and shows

$$
\psi_{1 B}-\psi_{2 B}=-\frac{F_{n B}}{k^{2} G_{x z}}\left(\frac{1}{A_{1}}+\frac{1}{A_{2}}\right)
$$


where $k^{2}$ is the through-thickness shear correction factor and is usually taken to be $5 / 6$ for isotropic materials, $G_{15}$ the though-thickness shear modulus, $A_{1}$ and $A_{2}$ the cross-sectional areas for beam 1 and 2, respectively. It is seen that when $F_{n, B}=0, D_{c o p}=0$. That is, both $F_{n, B}=0$ and $D_{o p}=0$ in the pure mode II modes $\varphi_{\beta_{1}}$ and $\varphi_{\beta_{2}}$. Moreover, both $F_{s B}=0$ and $D_{s h}=0$ in the pure mode I modes $\varphi_{\theta_{1}}$ and $\varphi_{\theta_{2}}$. Consequently, there is no interaction between the pure mode I modes, i.e. $\varphi_{\theta_{1}}$ and $\varphi_{\theta_{2}}$, and the pure mode II modes, i.e. $\varphi_{\beta_{1}}$ and $\varphi_{\beta_{2}}$, in Timoshenko beam theory and Eq. (10) is the correct partition for a mixed mode. That is in Timoshenko beam theory

$$
G_{I}=\alpha_{\theta_{1}}^{2} G_{\theta_{1}} \text { and } G_{I I}=G-G_{I}
$$

It is worth noting that crack tip running contact will occur at the pure mode II modes $\varphi_{\beta_{1}}$ and $\varphi_{\beta_{2}}$ as $D_{o p}=0$. Therefore, a pure mode II region is generated beyond $\varphi_{\beta_{1}}$ or $\varphi_{\beta_{2}}$.

Now, two crack tip shear forces $P_{1 B}$ and $P_{2 B}$ are included in Timoshenko beam theory. Since the through-thickness shear effect does not generate any axial displacement, $P_{1 B}$ and $P_{2 B}$ produce pure mode I fracture only. Here, it is designated as $P$ mode I. The relative crack tip opening displacement $D_{o p P}$ and normal force $F_{n B P}$ are easily obtained.

$$
\begin{gathered}
D_{b B P}=\frac{\left(\gamma P_{1 B}-P_{2 B}\right) \delta a}{\gamma b h_{1} k^{2} G_{x}} \\
F_{n B P}=\frac{\gamma P_{1 B}-P_{2 B}}{1+\gamma}
\end{gathered}
$$

The energy release rate $G_{P}$ in $P$ mode $I$ is then determined by using the crack closure technique.

$$
G_{P}=\frac{\left(\gamma P_{1 B}-P_{2 B}\right)^{2}}{2 b^{2} h k_{1}^{2} G_{x z} \gamma(1+\gamma)}
$$

It is easy to show that $G_{P}$ above is equal to

$$
G_{P}=\frac{1}{2 b k^{2} G_{I Z}}\left[\frac{P_{1 B}^{2}}{A_{1}}+\frac{P_{2 B}^{2}}{A_{2}}-\frac{\left(P_{1 B}+P_{2 B}\right)^{2}}{A}\right]
$$

Similarly, there is no stealthy interaction between the $P$ mode I and the pure mode II modes $\varphi_{\beta_{1}}$ and $\varphi_{\beta_{2}}$. However, there is modal coupling between the $P$ mode I and pure mode I $\varphi_{\theta_{1}}$. This modal coupling is the sum of work done by the crack tip normal force $F_{n B}$ in $\varphi_{\theta_{1}}$ mode I on the relative crack tip opening displacement $D_{o p P}$ in $P$ mode I and work done by the crack tip normal force $F_{n B P}$ in $P$ mode I on the relative crack tip opening displacement $D_{o p}$ in $\varphi_{\theta_{1}}$ mode I when fracture occurs. In the same way as $\Delta G_{\theta_{1} \beta_{1}}$ in Eq. (15) and $\Delta G_{\theta_{1} \beta_{2}}$ in Eq. (16) were determined, the coupling is found to be 


$$
\Delta G_{\theta_{1} P}=\frac{4 \sqrt{3}\left(\gamma P_{1 B}-P_{2 B}\right)}{b^{2} h_{1}^{2}(1+\gamma)\left(k^{2} G_{x z} E\right)^{1 / 2}}
$$

The total energy release rate becomes

$$
G=\alpha_{\theta_{1}}^{2} G_{\theta_{1}}+\alpha_{\beta_{1}}^{2} G_{\beta_{1}}+\alpha_{\beta_{2}}^{2} G_{\beta_{2}}+\alpha_{\beta_{1}} \alpha_{\beta_{2}} G_{\beta_{1} \beta_{2}}+G_{P}+\alpha_{\theta_{1}} \Delta G_{\theta_{1} P}
$$

A mixed mode is partitioned as

$$
G_{I}=\alpha_{\theta_{1}}^{2} G_{\theta_{1}}+G_{P}+\alpha_{\theta_{1}} \Delta G_{\theta_{1} P} \text { and } G_{I I}=G-G_{I}
$$

So far, mixed mode partition theories have been developed in both Euler and Timoshenko beam theories. It is seen that full stealthy interaction occurs in the former while there is no interaction in the latter. An averaged partition theory can be achieved by halving the interactions. That is

$$
G_{I}=\alpha_{\theta_{1}}^{2} G_{\theta_{1}}+1 / 2 \alpha_{\theta_{1}} \alpha_{\beta_{1}} \Delta G_{\theta_{\beta_{1}}}+1 / 2 \alpha_{\theta_{1}} \alpha_{\beta_{2}} \Delta G_{\theta_{\beta_{2}}}+G_{P}+\alpha_{\theta_{1}} \Delta G_{\theta_{1} P} \text { and } G_{I I}=G-G_{I}
$$

It is worth noting that crack tip running contact will occur at locations different from the pure mode II modes $\varphi_{\beta_{1}}$ or $\varphi_{\beta_{2}}$ due to the addition of through-thickness shear opening displacement $D_{o p p}$.

\subsection{Laminated composite double cantilever beams}

The DCB in Fig. 2 is now considered to be a composite laminate. Detailed development of this section can be found in [21]. Here, the essential part is derived. The use of beam theory means that only the $A_{11}, B_{11}$ and $D_{11}$ laminate stiffness coefficients from classical lamination theory (CLT) are required. The 11 subscripts are therefore discarded in the present notation. By considering the strain energy, the energy release rate $G$ at the crack tip point B is

$$
\begin{aligned}
G= & \frac{1}{b} \frac{\partial U}{\partial a}=\frac{1}{2 b^{2}}\left(\frac{M_{1 B}^{2}}{D_{1}^{*}}+\frac{M_{2 B}^{2}}{D_{2}^{*}}-\frac{M_{B}^{2}}{D^{*}}+\frac{N_{1 B}^{2}}{A_{1}^{*}}+\frac{N_{2 B}^{2}}{A_{2}^{*}}-\frac{N_{B}^{2}}{A^{*}}\right. \\
& \left.-\frac{2 B_{1} M_{1 B} N_{1 B}}{B_{1}^{*}}-\frac{2 B_{2} M_{2 B} N_{2 B}}{B_{2}^{*}}+\frac{2 B M_{B} N_{B}}{B^{*}}\right) \\
= & \frac{1}{2}\left\{\begin{array}{llll}
M_{1 B} & M_{2 B} & N_{1 B} & N_{2 B}
\end{array}\right\}[C]\left\{\begin{array}{llll}
M_{1 B} & M_{2 B} & N_{1 B} & N_{2 B}
\end{array}\right\}^{T}
\end{aligned}
$$

where

$$
\begin{gathered}
A_{i}^{*}=A_{i}-B_{i}^{2} / D_{i} \text { and } B_{i}^{*}=B_{i}^{2}-A_{i} D_{i} \text { and } D_{i}^{*}=D_{i}-B_{i}^{2} / A_{i} \\
N_{B}=N_{1 B}+N_{2 B} \text { and } M_{B}=M_{1 B}+M_{2 B}+\frac{1}{2}\left(h_{1} N_{2 B}-h_{i 2} N_{1 B}\right)
\end{gathered}
$$

Three independent pure mode I modes $\varphi_{\theta_{1}}, \varphi_{\theta_{2}}$ and $\varphi_{\theta_{3}}$ can be obtained from the condition $D_{\text {sh }}=0$. By using orthogonal condition three independent pure mode II modes $\varphi_{\beta_{1}}, \varphi_{\beta_{2}}$ and $\varphi_{\beta_{3}}$ 
can also be achieved which correspond to $F_{n B}=0$. By using $\varphi_{0_{1}}, \varphi_{\beta_{1}}, \varphi_{\beta_{2}}$ and $\varphi_{\beta_{3}}$ for the mixed mode partition, the mode partition coefficients are found to be

$$
\left\{\begin{array}{c}
\alpha_{\theta_{1}} \\
\alpha_{\beta_{1}} \\
\alpha_{\beta_{2}} \\
\alpha_{\beta_{3}}
\end{array}\right\}=\left\{\begin{array}{c}
\frac{M_{2 B} \beta_{2}+N_{1 B} \beta_{1}-M_{1 B} \beta_{1} \beta_{2}}{\beta_{2}\left(\theta_{1}-\beta_{1}\right)}+\frac{N_{2 B} \beta_{1}}{\beta_{3}\left(\theta_{1}-\beta_{1}\right)} \\
\frac{M_{1 B} \theta_{1} \beta_{2}-M_{2 B} \beta_{2}-N_{1 B} \theta_{1}}{\beta_{2}\left(\theta_{1}-\beta_{1}\right)}-\frac{N_{2 B} \theta_{1}}{\beta_{3}\left(\theta_{1}-\beta_{1}\right)} \\
\frac{N_{1 B}}{\beta_{2}} \\
\frac{N_{2 B}}{\beta_{3}}
\end{array}\right\}
$$

where

$$
\begin{gathered}
\theta_{1}=\frac{\left(B_{2}^{2}-A_{2} D_{2}\right)\left(B_{1}+h_{1} A_{1} / 2\right)}{\left(B_{1}^{2}-A_{1} D_{1}\right)\left(B_{2}-h_{2} A_{2} / 2\right)} \\
\theta_{2}=-\frac{B_{1}+h_{1} A_{1} / 2}{D_{1}+h_{1} B_{1} / 2} \\
\theta_{3}=\frac{\left(B_{1}+h_{1} A_{1} / 2\right)\left(B_{2}^{2}-A_{2} D_{2}\right)}{\left(D_{2}-h_{2} B_{2} / 2\right)\left(B_{1}^{2}-A_{1} D_{1}\right)} \\
\beta_{2}=\frac{\beta_{1}=-\frac{D_{2}^{*}\left(D_{1}^{*}+D_{1}^{*} \theta_{1}-D^{*}\right)}{D_{1}^{*}\left(D_{2}^{*}+D_{2}^{*} \theta_{1}-D^{*} \theta_{1}\right)}}{\theta_{2}\left(\frac{B h_{2}}{B^{*}}-\frac{1}{A_{1}^{*}}+\frac{1}{A^{*}}+\frac{h_{2}}{2 D^{*}}-\frac{B_{1}}{B_{1}^{*}}+\frac{B_{2}^{2}}{B^{*}}\right)+\frac{1}{D^{*}}-\frac{1}{D^{*}}} \frac{h_{2}}{2 D^{*}}+\frac{B_{1}}{B_{1}^{*}}-\frac{B}{B^{*}} \\
\beta_{3}=\frac{\theta_{3}\left(\frac{h_{1}}{2 D^{*}}-\frac{B}{B^{*}}\right)-\frac{1}{D_{1}^{*}}+\frac{1}{D^{*}}}{\theta_{3}\left(\frac{B h_{1}}{B^{*}}+\frac{1}{A_{2}^{*}}-\frac{1}{A^{*}}-\frac{h_{1}^{2}}{4 D^{*}}\right)-\frac{h_{1}}{2 D^{*}}+\frac{B}{B^{*}}}
\end{gathered}
$$

In the context of Euler beam theory, the mode I energy release rate is then calculated as

$$
G_{I}=\alpha_{\theta_{1}}^{2} G_{\theta_{1}}+\alpha_{\theta_{1}} \alpha_{\beta_{1}} \Delta G_{\theta_{1} \beta_{1}}+\alpha_{\theta_{1}} \alpha_{\beta_{2}} \Delta G_{\theta_{1} \beta_{2}}+\alpha_{\theta_{1}} \alpha_{\beta_{3}} \Delta G_{\theta_{1} \beta_{3}}
$$

where 


$$
\begin{gathered}
G_{\theta_{1}}=\frac{1}{2 b^{2}}\left[\frac{1}{D_{1}^{*}}+\frac{\theta_{1}^{2}}{D_{2}^{*}}-\frac{\left(1+\theta_{1}\right)^{2}}{D^{*}}\right] \\
\Delta G_{\theta_{1} \beta_{1}}=\frac{F_{n i B} \delta a}{4 b^{2}}\left(\frac{1}{D_{1}^{*}}-\frac{\beta_{1}}{D_{2}^{*}}\right) \\
\Delta G_{\theta_{1} \beta_{2}}=\frac{F_{n, D} \delta a}{4 b^{2}}\left(\frac{1}{D_{1}^{*}}-\frac{B_{1} \beta_{2}}{B_{1}^{*}}\right) \\
\Delta G_{\theta_{1} \beta_{3}}=\frac{F_{n, B} \delta a}{4 b^{2}}\left(\frac{1}{D_{1}^{*}}+\frac{B_{2} \beta_{3}}{B_{2}^{*}}\right)
\end{gathered}
$$

and

$$
F_{n B} \delta a=\frac{2\left(D_{1}^{*} D^{*} \theta_{1}^{2}+D_{2}^{*} D^{*}-D_{1}^{*} D_{2}^{*}-2 D_{1}^{*} D_{2}^{*} \theta_{1}-D_{1}^{*} D_{2}^{*} \theta_{1}^{2}\right)}{D^{*}\left(D_{2}^{*}-D_{1}^{*} \theta_{1}\right)}
$$

Within the context of Timoshenko beam theory the last three terms in Eq. (39) disappear. This is for the reasons described in Section 2.1.

Now the through-thickness shearing effect under Timoshenko beam theory is accounted for. The only laminate shearing stiffness required for beams is $A_{55}$ from CLT. This quantity is represented by $H$ in the present notation. By considering the additional opening displacement, the normal force at the crack tip due to shear forces and the interaction of these quantities with the $\varphi_{0}$ mode I, the following is obtained:

$$
\begin{gathered}
G=\frac{1}{2 b^{2}}\left(\frac{M_{1 B}^{2}}{D_{1}^{*}}+\frac{M_{2 F}^{2}}{D_{2}^{*}}-\frac{M_{B}^{2}}{D^{*}}+\frac{N_{1 F}^{2}}{A_{1}^{*}}+\frac{N_{2 F}^{2}}{A_{2}^{*}}-\frac{N_{B}^{2}}{A^{*}}\right. \\
\left.-\frac{2 B_{1} M_{1 B} N_{1 B}}{B_{1}^{*}}-\frac{2 B_{2} M_{2 B} N_{2 B}}{B_{2}^{*}}+\frac{2 B M_{B} N_{B}}{B^{*}}\right)+G_{F^{*}}+2 \alpha_{\theta_{1}} \Delta G_{\theta_{1} P} \\
G_{I}=\alpha_{\theta_{1}}^{2} G_{\theta_{1}}+G_{P}+2 \alpha_{\theta_{1}} \Delta G_{\theta_{1} P}
\end{gathered}
$$

where

$$
\begin{gathered}
G_{P}=\frac{\left(H_{1} P_{2 B}-H_{2} P_{1 B}\right)^{2}}{2 b^{2} H_{1} H_{2}\left(H_{1}+H_{2}\right)} \\
\Delta G_{\theta_{1} P}=\frac{1}{2 b^{2}}\left(\frac{P_{1 B}}{H_{1}}-\frac{P_{2 B}}{H_{2}}\right)\left[\frac{H_{1} H_{2}}{2\left(H_{1}+H_{2}\right)}\left(\frac{1}{D_{1}^{*}}+\frac{\theta_{1}^{2}}{D_{2}^{*}}-\frac{\left(1+\theta_{1}\right)^{2}}{D^{*}}\right)\right]^{1 / 2}
\end{gathered}
$$

Obviously there is no interaction between the $P$ mode I mode and pure mode II modes in Eq. (46). 


\section{Clamped-clamped isotropic beams}

A general clamped-clamped beam with a fracture is shown in Fig. 3. Contact between the upper and lower beams is not considered. Crack tip forces and moments can be determined analytically in terms of the applied forces $P_{1}$ and $P_{2}$. A mixed mode can be readily partitioned using the theory for isotropic DCB in Section 2.1. As the expressions of the crack tip forces and moments are in complex forms, they are not presented here. Fortunately, the pure modes in terms of $P_{1}$ and $P_{2}$ are relatively simple and will be derived below.

In the same way as in Section 2.1, two sets of pure modes are expected. The first set corresponds to zero crack tip relative shearing displacement (pure mode I) $D_{c h}=0$ and zero crack tip normal force (pure mode II) $F_{n B}=0$. The second set corresponds to zero relative opening displacement (pure mode II) $D_{o p}=0$ and zero crack tip shearing force (pure mode I) $F_{s E}=0$.

The study is given in the context of Euler beam theory first. The left crack tip is the one considered. The right one has the same set of pure modes as those of the left one in this particular case. The relative crack tip shearing displacement $D_{\text {sh }}$ is expressed as

$$
D_{s h}=\left(\bar{u}_{1}\right)_{x=\delta n}-\left(\bar{u}_{2}\right)_{x=\delta \phi t}=\left(u_{1}\right)_{x=\delta n}+\frac{h_{1}}{2}\left(\frac{d w_{1}}{d x}\right)_{x=\delta n}-\left(u_{2}\right)_{x=\delta n}+\frac{h_{2}}{2}\left(\frac{d w_{2}}{d x}\right)_{x=\delta a}
$$

where $\bar{u}$ represents the axial displacement at the interface. $D_{s h}=0$ gives

$$
\frac{P_{2}}{P_{1}}=\theta_{P}=-\frac{\gamma^{2}\left(C_{1} \gamma^{2}-C_{2} \gamma+C_{3}\right)}{C_{3} \gamma^{2}-C_{2} \gamma+C_{1}}
$$

where

$$
\begin{gathered}
C_{1}=2 x_{p}\left(a-x_{p}\right)^{2}\left(a+L_{1}+L_{2}\right)^{3} \\
C_{2}=2 x_{p}\left(a-x_{p}\right)^{2}\left(C_{7}+3 a^{2}\left(L_{1}+L_{2}\right)-2 a^{3}\right) \\
C_{3}=2 a^{3} x_{p}^{3}-a x_{p}^{2}\left(C_{7}+6 a^{2} L_{2}+4 a^{3}\right)+2 a^{2} x_{p}\left(C_{7}-3 a L_{1} L_{2}+3 a^{2} L_{2}+a^{3}\right)-a C_{8} \\
C_{7}=\left(L_{1}+L_{2}\right)^{3}+3 a\left(L_{1}+L_{2}\right)^{2} \\
C_{8}=a^{2} L_{1}^{2}\left(L_{1}+3 L_{2}+3 a\right)
\end{gathered}
$$

The orthogonal condition to the zero relative shearing displacement condition $D_{s h}=0$ is zero crack tip normal force condition $F_{n B}=0$. The mode corresponding to this condition could be derived by applying the orthogonality condition, however it is more convenient in this instance to simply enforce $F_{n B}=0$. The crack tip normal force is calculated as

$$
F_{n B}=\alpha_{\theta_{1}} F_{n B \theta_{1}}+\alpha_{\beta_{1}} F_{n B \beta_{1}}+\alpha_{\beta_{2}} F_{n B B_{2}}=\alpha_{\theta_{1}} F_{n B \theta_{1}}
$$


The mode II condition itself dictates that the contribution to the normal force from modes and $\varphi_{\beta_{2}}$ is zero. Therefore the requirement is simply $\alpha_{\theta_{1}}=0$ which gives

$$
\frac{P_{2}}{P_{1}}=\beta_{F^{\prime}}=\frac{\gamma^{2}\left(C_{1} \gamma^{3}+C_{1} \gamma^{2}+C_{5} \gamma+C_{6}\right)}{C_{6} \gamma^{3}+C_{5} \gamma^{2}+C_{4} \gamma+C_{1}}
$$

where

$$
\begin{gathered}
C_{1}=2 x_{p}\left(a-x_{p}\right)^{2}\left(2 C_{7}+6 a^{2}\left(L_{1}+L_{2}\right)+5 a^{3}\right) \\
C_{5}=x_{p}^{3}\left(2 C_{7}+6 a^{2}\left(L_{1}+L_{2}\right)+14 a^{3}\right)-a x_{p}^{2}\left(C_{7}+12 a^{2} L_{1}-6 a^{2} L_{2}+28 a^{3}\right) \\
-2 a^{2} x_{p}\left(2 C_{7}-3 a^{2} L_{1}+6 a^{2} L_{2}-7 a^{3}-9 a L_{1} L_{2}\right)+3 a C_{8} \\
C_{6}=6 a^{3} x_{p}^{3}-3 a x_{p}^{2}\left(C_{7}+6 a^{2} L_{2}+4 a^{3}\right)+6 a^{2} x_{p}\left(C_{7}-3 a L_{1} L_{2}+3 a^{2} L_{2}+a^{3}\right)-3 a C_{8}
\end{gathered}
$$

It is worth noting that $\theta_{P}=\theta_{1}$ in Eq. (6) and $\beta_{P}=\beta_{1}$ in Eq. (7) for a symmetric crack with midspan loads. Now considering the second set of pure modes, the pure mode I mode is given by zero crack tip shear force $F_{s B}=0 . F_{s B}$ is given by

$$
F_{s B}=\alpha_{0_{1}} F_{s B 0_{1}}+\alpha_{B_{1}} F_{s B B_{1}}+\alpha_{B_{2}} F_{s B B_{2}}
$$

The mode partition coefficients and the modal crack tip shear forces are known from previous work [20]. Setting $F_{s B}=0$ gives

$$
\frac{P_{2}}{P_{1}}=\theta_{P}^{\prime}=\theta_{E}^{\prime}=-1
$$

Finally, for the pure mode II mode from the second set, the relative crack tip opening displacement $D_{o p}$ is zero. This condition gives

$$
\frac{P_{2}}{P_{1}}=\beta_{P}^{\prime}=\beta_{E}^{\prime}=\gamma^{3}
$$

That $\theta_{P}^{\prime}$ and $\beta_{P}^{\prime}$, relating $P_{1}$ and $P_{2}$, are the same as $\theta_{E}^{\prime}$ and $\beta_{F}^{\prime}$ given in Eqs. (13) and (14), which relate $M_{2 B}$ to $M_{1 B}$, should be no surprise since the axial forces $N_{1 B}$ and $N_{2 B}$ induced at the crack tip by $P_{1}$ and $P_{2}$ have no effect on the opening displacement. Also, if $P_{1}$ and $P_{2}$ are equal and opposite $P_{2} / P_{1}=-1$, then regardless of how beams 1 and 2 deflect, beams 3 and 4 remain undeflected and the crack tip rotations are zero. Therefore $N_{1 B}$ and $N_{2 B}$ are both zero and the two crack tip bending moments are in the ratio of $\theta_{E}^{\prime}$. If $P_{1}$ and $P_{2}$ are applied in different locations then this would not be observed because each load would have a different moment arm around the crack tip.

Within the context of Timoshenko beam theory, the expressions for $P_{2} / P_{1}=\theta_{P}$ pure mode I and $P_{2} / P_{1}=\beta_{P}$ pure mode II are not as simple as those in Eqs. (50) and (57). However when the 
through-thickness shear effect is not excessively large, Eqs. (50) and (57) are good approximations. A region of pure mode II fracture occurs beyond $P_{2} / P_{1}=\beta_{P}$.

\section{Clamped-clamped composite laminated beams}

A clamped-clamped composite laminated beam with a symmetric delamination is considered. The loads $P_{1}$ and $P_{2}$ are applied at the mid-span. Again, contact is not considered. The study is again carried out using Euler beam theory first. In the same way as in the study in Section 3, $D_{s h}=0$ gives pure mode I condition

$$
\frac{P_{2}}{P_{\perp}}=\theta_{P}=-\frac{B_{2}^{*}\left(2 B_{1}+h_{1} A_{1}\right)}{B_{1}^{*}\left(2 B_{2}-h_{2} A_{2}\right)}
$$

Pure mode II $P_{2} / P_{1}=\beta_{p}$ arising from $F_{n, 3}=0$ is too complex to be presented here algebraically. $D_{o p}=0$ gives pure mode II.

$$
\frac{P_{2}}{P_{1}}=\beta_{P}^{\prime}=\beta_{E}^{\prime}=\frac{D_{2}^{*}}{D_{1}^{*}}
$$

As expected its orthogonal pure mode I is easily obtained as

$$
\frac{P_{2}}{P_{1}}=\theta_{P}^{\prime}=\theta_{E}^{\prime}=-1
$$

Similarly, within the context of Timoshenko beam theory, the expressions for $P_{2} / P_{1}=\theta_{P}$ pure mode I and $P_{2} / P_{1}=\beta_{P}$ pure mode II are too complex to be presented here algebraically. However, when the through-thickness shear effect is not excessively large, they are very close to those in Euler beam theory.

\section{Clamped circular isotropic plate}

A clamped circular plate with a central delamination and loads is shown in Fig. 4. By using the theory in the previous sections, the first set of pure modes in Euler beam theory are found to be

$$
\frac{P_{2}}{P_{1}}=\theta_{p}=\theta_{1}
$$

and

$$
\frac{P_{2}}{P_{1}}=\beta_{p}=\beta_{1}
$$

where $\theta_{1}$ and $\beta_{1}$ are given in Eqs. (6) and (7), respectively. The corresponding energy release rates are given by Eqs. (69) and (70). 


$$
\begin{gathered}
G_{\theta_{\rho}}=\frac{3 P_{1}^{2} \gamma\left(1-\nu^{2}\right)}{2 E h_{1}^{3} \pi^{2}(1+\gamma)} \\
G_{\beta_{\rho}}=\frac{9 P_{1}^{2} \gamma\left(1-\nu^{2}\right)(1+\gamma)}{2 E h_{1}^{3} \pi^{2}(1+3 \gamma)^{2}}
\end{gathered}
$$

The second set of pure mode I and II modes are the same as those in Eqs. (62) and (63). In Timoshenko beam theory, the first set of pure modes is approximately pure and the second set disappears.

\section{Numerical investigations}

To validate the theory, an FEM simulation capability was developed based on Euler and Timoshenko beam theory and 2D elasticity. Normal and shear point interface springs with very high stiffness were used. The energy release rate partition was calculated using the virtual crack closure technique in conjunction with these interface springs [22, 23]. A contact algorithm was also implemented to deal with any possible contact in loading.

\subsection{Tests with double cantilever beams}

Two DCB cases were investigated. The first case is an isotropic one, the data for which is given in Table 1. Since normalised data is used in this case, an interface spring stiffness of $10^{6}$ was sufficient to model perfectly bonded plies. The second case is an orthotropic laminated composite one with real data. The material properties are for a T300/976 graphite/epoxy ply [23]. It has a cross-ply lay-up with 24 plies. In this case, an interface spring stiffness of $10^{14} \mathrm{~N} / \mathrm{m}$ was required. There is a delamination between the 16th and 17th plies. The data for this case is given in Table 2. Present theoretical and numerical results are presented in Fig. 5 and Table 3 for the isotropic DCB and in Fig. 6 and Table 4 for the composite DCB.

Three sets of numerical simulations were run using FEM. The first set, which used linear Timoshenko beam elements, is for comparison with the present Euler partition rule. Very large out-of-plane shear moduli $\left(G_{x z}=10^{4}\right.$ and $G_{13}=G_{23}=10^{14} \mathrm{~N} / \mathrm{m}^{2}$ for the isotropic and laminated composite case respectively) were used to simulate Euler beam theory. Two layers of elements were used to represent the beams with one on either side of the fracture. The elements were distributed uniformly. To avoid shear locking, reduced integration was applied. Use of linear Timoshenko beams correctly enforces continuity along the interface ahead of the crack tip. A very good agreement is observed between present theoretical and numerical predictions for both DCBs.

The second set of simulations, which was the same as the first set but which instead used the normal out-of-plane shear moduli (those given in Tables 1 and 2) and a shear correction factor of $k^{2}=5 / 6$, is compared against the present Timoshenko partition rule. Again, a very good agreement is observed.

The final simulations used QUAD4 elements with the normal out-of-plane shear moduli. In the isotropic case, two and four layers of QUAD4 elements were needed in the top and bottom beams respectively for sufficient convergence. In the composite case, a layer of QUAD4 elements was used for each individual ply. This was found to be necessary to obtain converged results. The elements were distributed uniformly along the length and thickness. The results from these simulations are compared against the present Euler, Timoshenko and averaged partition 
rules. In general, QUAD4 results agree well with the averaged partition rule. Note that a pure mode II region starts around $N_{2}=-15 \mathrm{kN}$ in Fig. 6 for the composite DCB due to crack tip running contact.

\subsection{Tests with clamped-clamped beams}

Two clamped-clamped beam cases were investigated. The first case is an asymmetric, isotropic one as shown in Fig. 3, the data for which is given in Table 5. The second case is a symmetric laminated composite one. It has a quasi-isotropic lay-up with 16 plies. There is a delamination between the 12th and 13th plies, which gives a thickness ratio of $\gamma=3$. The data for this case is given in Table 6 with the material properties given in Table 2. The material properties are for a T300/976 graphite/epoxy ply [23]. In both cases an interface spring stiffness of $10^{14} \mathrm{~N} / \mathrm{m}$ was used. Present theoretical and numerical results are presented in Fig. 7 and Table 7 for the isotropic beam and in Fig. 8 and Table 8 for the composite beam.

The test procedure is identical to that for DCBs in Section 6.1. One set of simulations, which used linear Timoshenko beam elements, is compared against the present Euler rule. Very large out-of-plane shear moduli $G_{x z}=G_{13}=G_{23}=10^{16} \mathrm{~N} / \mathrm{m}^{2}$ were used to simulate Euler beam theory. An excellent agreement is observed. Another set of simulations, which was the same as the first set but which instead used the normal out-of-plane shear moduli (those given in Tables 5 for isotropic beam and Table 2 for composite beam) and a shear correction factor of $k^{2}=5 / 6$, is compared against the present Timoshenko rule. Again, the agreement is good. The final simulations used QUAD4 elements with the normal out-of-plane shear moduli. In the composite case, a layer of QUAD4 elements was used for each individual ply. In the isotropic case, two and four layers of QUAD4 elements were needed in the top and bottom beams respectively. In general, QUAD4 results agree well with the averaged partition rule. Note that pure mode II region starts beyond pure mode II $\beta_{P}=2.8106$ for the isotropic beam and $\beta_{P}=4.9836$ for the composite beam due to crack tip running contact.

\subsection{Tests with clamped circular isotropic plates}

Table 9 gives the data of a circular plate shown in Fig. 4. The loads are set to be $P_{1}=1 \mathrm{~N}$ and $P_{2}=\theta_{P} P_{1}=-4 \mathrm{~N}$ for $\theta_{P}$ mode I and $P_{2}=\beta_{P} P_{1}=20 / 7 \mathrm{~N}$ for $\beta_{P}$ mode II. Table 10 records the results from Eqs. (69) and (70) and circular ring FEM simulations with $G_{x z}=10^{16} \mathrm{~N} / \mathrm{m}^{2}$ and $k_{s}=10^{14} \mathrm{~N} / \mathrm{m}$. Excellent agreement is again observed between the analytical and numerical results.

\section{Conclusions}

Completely analytical theories are developed for the mixed mode partition of one-dimensional fracture in layered isotropic and laminated composite DCBs. The theories are readily extended to general composite beams and circular plates. Two sets of orthogonal pure fracture modes are discovered which are distinct from each other in Euler beam or plate theory and coincide at the Wang-Harvey set in Timoshenko beam or plate theory. The Wang-Harvey set forms a unique complete orthogonal pure mode basis within the contexts of both Euler and Timoshenko beam or plate theories. A mixed mode is successfully partitioned by using Wang-Harvey set. Stealthy interactions are revealed between the Wang-Harvey pure mode I modes and mode II modes in 
Euler beam theory. Although the stealthy interactions do not generate any net amount of energy release rate, they do cause energy flow between pure modes and alter the partitions of a mixed mode. The present Euler rule and Timoshenko rule agree very well with the respective beam FEM predictions. The present averaged rule agrees well with the 2D FEM predictions.

The developed theories can provide academic researchers with theoretical tools for determining critical energy release rates of pure modes and developing fracture propagation criteria. Moreover, they can provide industrial engineers with analytical guidelines for design against fracture.

\section{References}

1. Williams JG. On the calculation of energy release rates for cracked laminates. International Journal of Fracture Mechanics 1988;36:101-19.

2. Schapery RA, Davidson BD. Prediction of energy release rate for mixed-mode delamination using classical plate theory. Applied Mechanics Review 1990;43:S281-7.

3. Hutchinson JW, Suo Z. Mixed mode cracking in layered materials. Advances in Applied Mechanics 1992;29:63-191.

4. Suo Z, Hutchinson JW. Interface ceack between two elastic layers. International Journal of Fracture Mechanics 1990;43:1-18.

5. Thouless MD, Evans AG, Ashby MF, Hutchinson JW. The edge cracking and saplling of brittle plates. Acta Metall 1987;35:1333-41.

6. Thouless MD. Fracture of a model interface under mixed mode loading. Acta Metall. Matter 1990;35:1135-40.

7. Chen J, Crisfield MA, Kinloch AJ, Busso EP, Matthews FL, Qiu Y. Predicting progressive delamination of composite material specimens via interface elements. Journal of Mechanics of Composite Materials and Structures 1999;6:301-17.

8. Chen J. Predicting progressive delamination of stiffened fibre-composite panel and repaired sandwich panel by decohesion models. Journal of Thermalplastic Composite Materials 2002;15:429-42.

9. Zou Z, Reid SR, Li S, Soden PD. General expressions for energy release rates for delamination in composite laminates. Proc. R. Soc. Lond. A 2002;458:645-67.

10. Wang J, Qiao P. Interface crack between two shear deformable elastic layers. Journal of the Mechanics and Physics of Solids 2004;52:891-905.

11. Li S, Reid SR, Zou Z. Modelling damage of multiple delaminations and transverse matrix cracking in laminated composites due to low velocity lateral impact. Composite Science and Technology 2005;66:827-36.

12. Nguyen C, Levy AJ. An exact theory of interfacial debonding in layered elastic composites. International Journal of solids and Structures 2009;46:2712-23.

13. Yan Y, Shang F. Cohesive zone modelling of interfacial delamination in PZT thin films. International Journal of solids and Structures 2009;46:2739-49.

14. Ouyang Z, Li G. Nonlinear interface shear fracture of end notched flexure specimens. International Journal of solids and Structures 2009;46:2659-68.

15. Wang S, Harvey C. Fracture mode partition rules for DCB. $17^{\text {th }}$ Interactional Conference on Composite/Nano Engineering (ICCE-17), July 2009, Honolulu, Hawaii, USA.

16. Harvey C, Wang S. Modelling of delamination propagation in composite laminated beam structures. Proceedings of the $7^{\text {th }}$ International Conference of Computational Methods in 
Science and Engineering (ICCMSE 2009), Simos T E (ed), American Institute of Physics, Rhodes, Greece.

17. Wang S, Harvey C. Mixed mode partition in one-dimensional fracture. Journal of Key Engineering Materials 2011;462-63:616-21. (Also, A plenary lecture in the $8^{\text {th }}$ International Conference on Fracture and Strength of Solids (FEOFS 2010), 7-9 ${ }^{\text {th }}$ June 2010, Kuala Lumpur, Malaysia)

18. Wang S, Guan L. On fracture mode partition theories, Computational Material Sciences 2011 (on line). (Also presented at the $20^{\text {th }}$ International Workshop on Computational Mechanics of Materials, September 2010, Loughborough University, UK.)

19. Wang S, Harvey C. A theory of one-dimensional fracture, A plenary lecture in the $16^{\text {th }}$ International Conference on Composite Structures (ICCS16), 28-30 ${ }^{\text {th }}$ June 2011, Porto, Portugal.

20. Wang S, Harvey C. Mixed mode partition theories for one-dimensional fracture. Engineering Fracture mechanics. (under review)

21. Harvey C, Wang S. Mixed mode partition theories for one-dimensional delamination in laminated composite beams. (in preparation)

22. Zhang Y, Wang S. Buckling, post-buckling and delamination propagation in debonded composite laminates Part 1 Theoretical development. Composite Structures 2009;88:121-30 (Also, a plenary lecture in the $16^{\text {th }}$ International Conference on Composite/Nano Engineering (ICCE-16), July 2008, Kunming, China).

23. Wang S, Zhang Y. Buckling, post-buckling and delamination propagation in debonded composite laminates Part 2 Numerical applications. Composite Structures 2009;88:131-46 (Also, a plenary lecture in the $16^{\text {th }}$ International Conference on Composite/Nano Engineering (ICCE-16), July 2008, Kunming, China). 


\section{Figure captions}

Figure 1: Some engineering structures exhibiting one-dimensional fracture.

Figure 2: A DCB with two tip bending moments and two tip axial forces.

Figure 3: A clamped-clamped beam with a fracture and its loading conditions.

Figure 4: A circular plate with a central delamination.

Figure 5: Comparison between various theories for isotropic DCB energy release rate partitions with varying $P_{1}\left(M_{1}=100, M_{2}=-200, N_{1}=100, N_{2}=0, P_{2}=0\right)$.

Figure 6: Comparison between various theories for laminated composite DCB energy release rate partitions with varying $N_{2}\left(M_{1}=1, M_{2}=0, N_{1}=0, P_{1}=0, P_{2}=0\right)$.

Figure 7: Comparison between various theories for clamped-clamped isotropic beam energy release rate partitions with varying $P_{2}$ and $P_{1}=1 \mathrm{~N}$.

Figure 8: Comparison between various theories for clamped-clamped composite laminated beam energy release rate partitions with varying $P_{2}$ and $P_{1}=1 \mathrm{~N}$. 


\section{Table captions}

Table 1: Data for numerical simulations of an isotropic DCB.

Table 2: Data for numerical simulations of a laminated composite DCB.

Table 3: Comparison between various theories for isotropic DCB energy release rate partitions with varying $P_{1}\left(M_{1}=100, M_{2}=-200, N_{1}=100, N_{2}=0, P_{2}=0\right)$.

Table 4: Comparison between various theories for laminated composite DCB energy release rate partitions with varying $N_{2}\left(M_{1}=1, M_{2}=0, N_{1}=0, P_{1}=0, P_{2}=0\right)$.

Table 5: Data for numerical simulations of a clamped-clamped isotropic beam

Table 6: Data for numerical simulations of a clamped-clamped composite laminated beam

Table 7: Comparison between various theories for clamped-clamped isotropic beam energy release rate partitions with varying $P_{2}$ and $P_{1}=1 \mathrm{~N}$.

Table 8: Comparison between various theories for clamped-clamped composite laminated beam energy release rate partitions with varying $P_{2}$ and $P_{1}=1 \mathrm{~N}$.

Table 9: Data of a clamped circular plate with a central fracture.

Table 10: Energy release rates $\left(\mu \mathrm{J} / \mathrm{m}^{2}\right)$ for $\theta_{P}$ and $\beta_{P}$ modes of the circular plate with a central crack. 


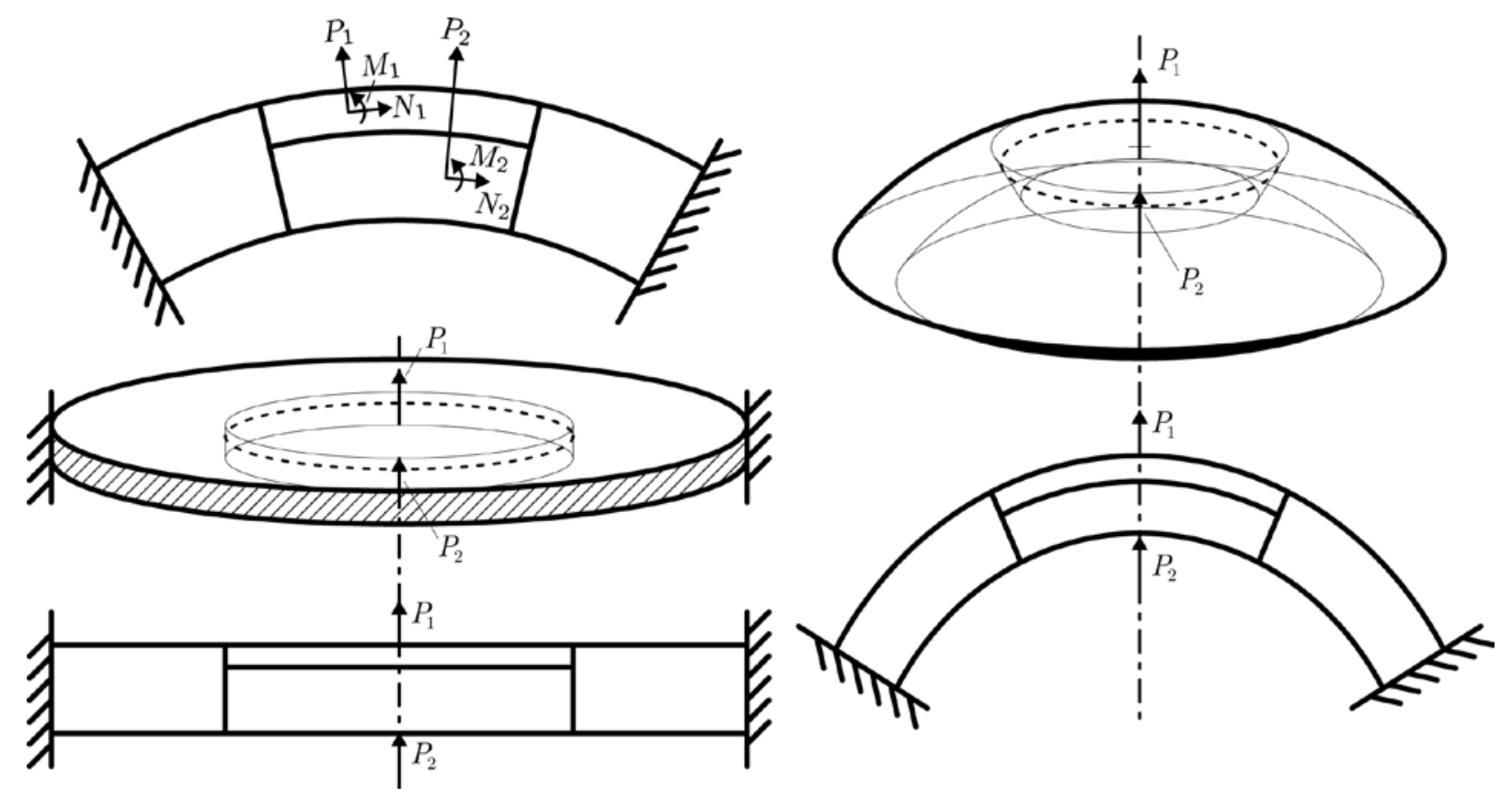

Figure 1: Some engineering structures exhibiting one-dimensional fracture. 


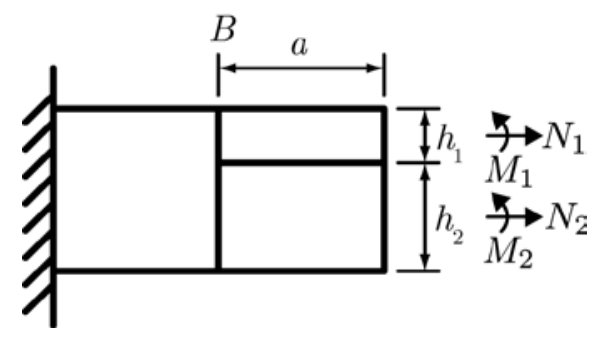

Figure 2: A DCB with two tip bending moments and two tip axial forces. 


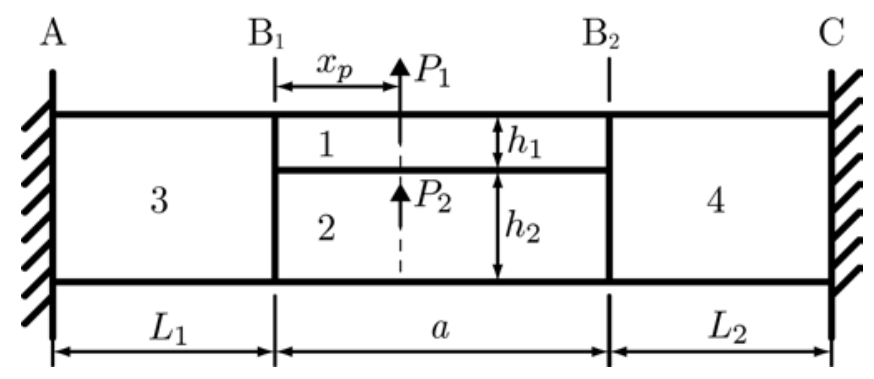

Figure 3: A clamped-clamped beam with a fracture and its loading conditions. 


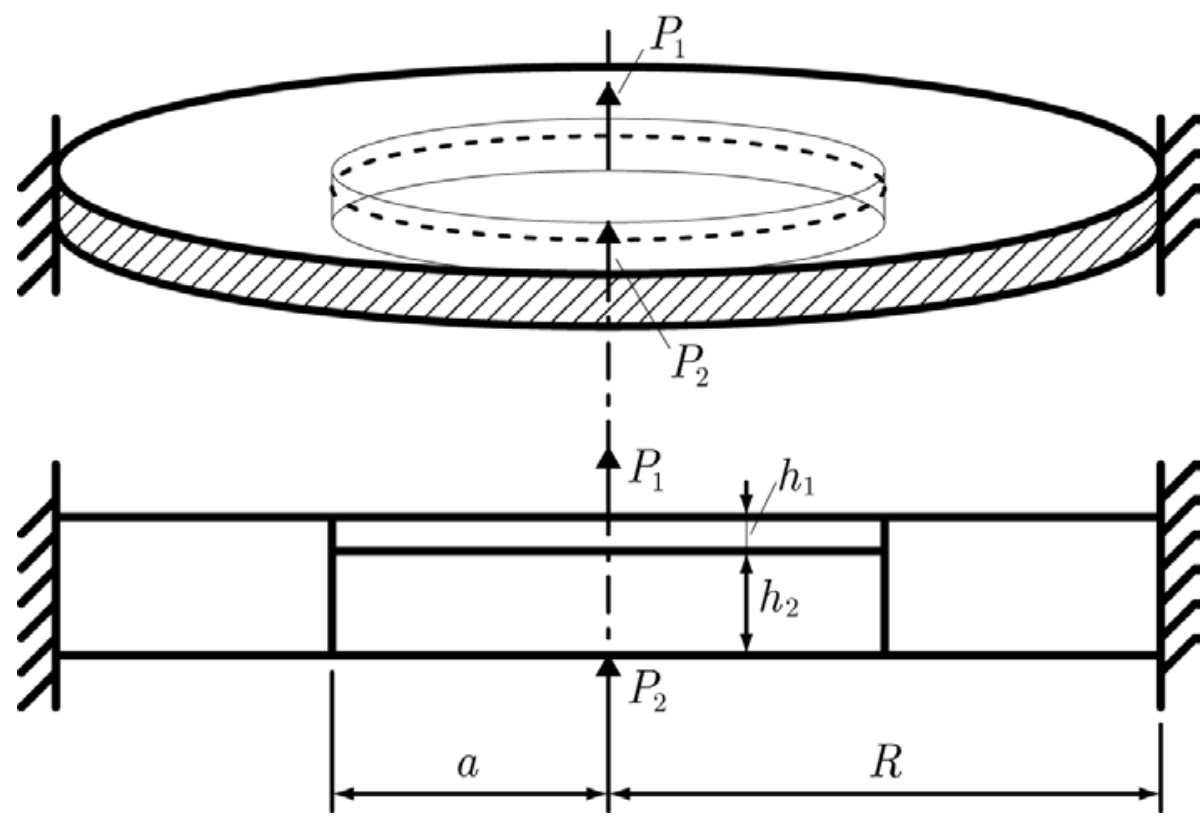

Figure 4: A circular plate with a central delamination. 


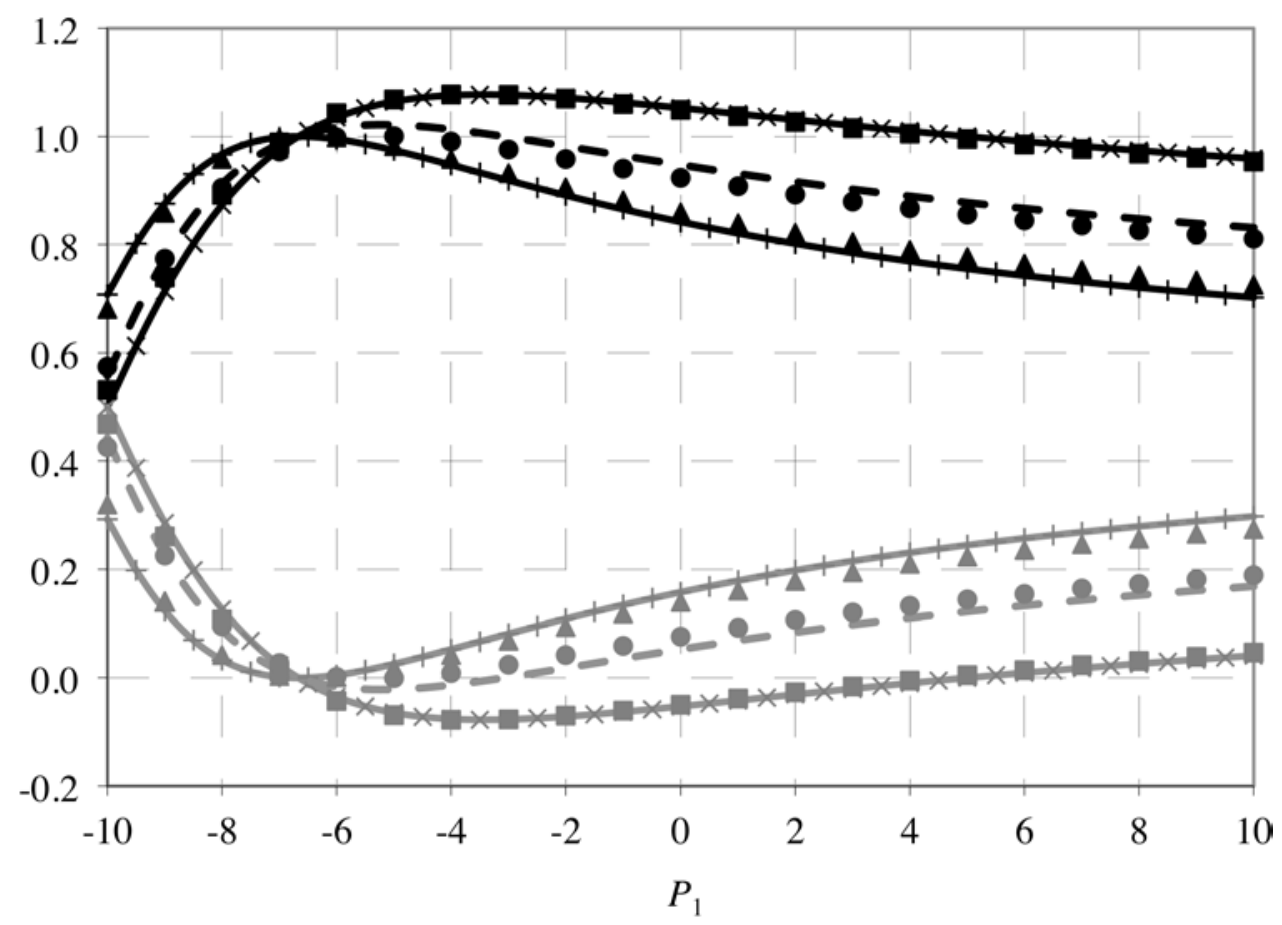

$$
\begin{aligned}
& \longrightarrow \text { Analytical Euler } G_{\mathrm{I}} / G \quad \longleftarrow \text { Analytical Euler } G_{\mathrm{II}} / G \\
& \text { - } 220 \times 2 \text { Numerical Euler } G_{\mathrm{I}} / G \quad 220 \times 2 \text { Numerical Euler } G_{\mathrm{II}} / G \\
& \text { - Analytical Timo. } G_{\mathrm{I}} / G \quad \text { Analytical Timo. } G_{\mathrm{II}} / G \\
& \Delta \quad 880 \times 2 \text { Numerical Timo. } G_{\mathrm{I}} / G \quad \Delta \quad 880 \times 2 \text { Numerical Timo. } G_{\mathrm{II}} / G \\
& \text { - Averaged Timo. \& Euler } G_{\mathrm{I}} / G \quad \text { - Averaged Timo. \& Euler } G_{\mathrm{II}} / G \\
& \text { - } 320 \times 62 \mathrm{D} \mathrm{FEM} G_{\mathrm{l}} / G \quad \text { - } 320 \times 62 \mathrm{D} \mathrm{FEM} G_{\mathrm{II}} / G
\end{aligned}
$$

Figure 5: Comparison between various theories for isotropic DCB energy release rate partitions with varying $P_{1}\left(M_{1}=100, M_{2}=-200, N_{1}=100, N_{2}=0, P_{2}=0\right)$. 


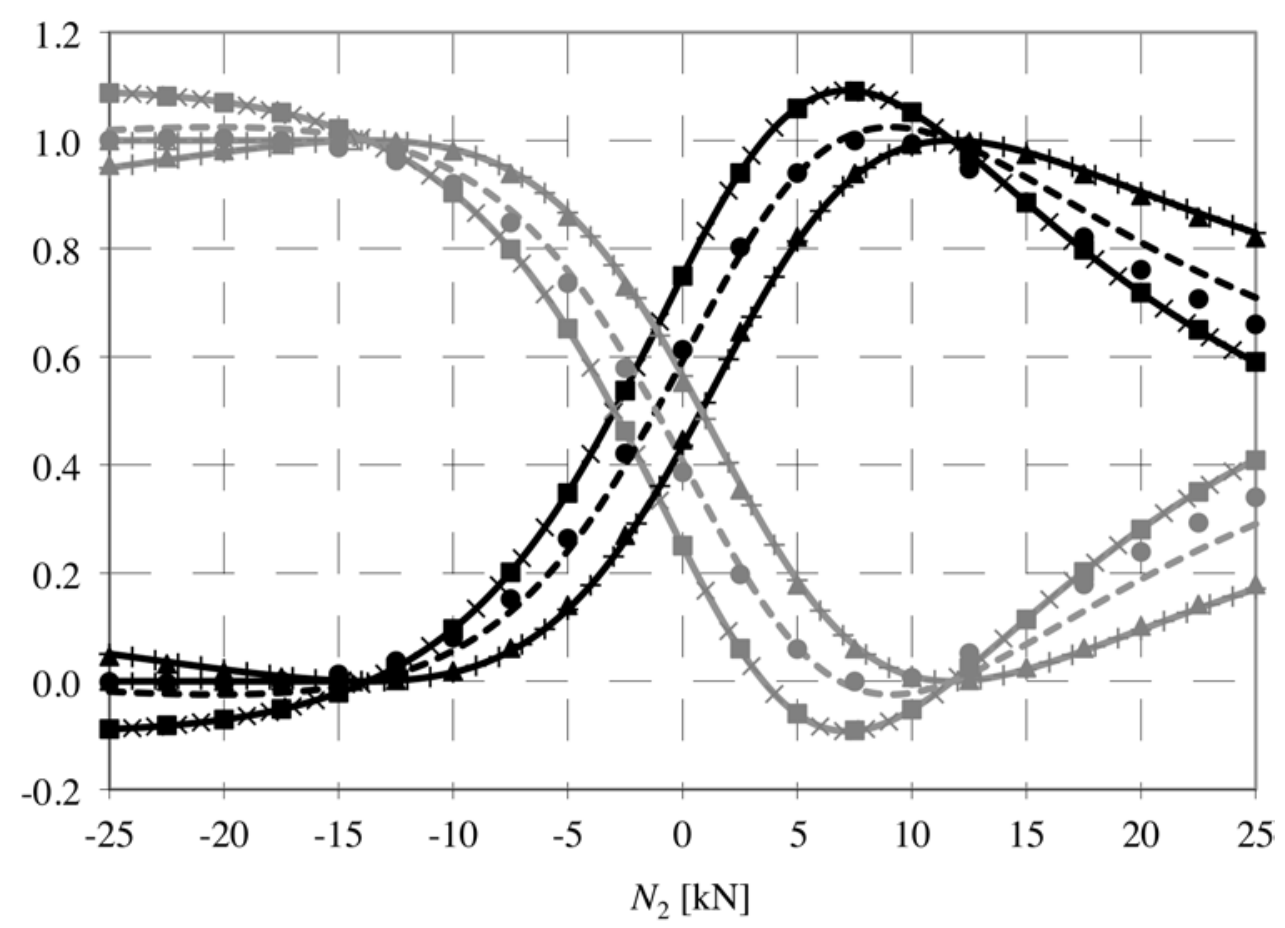

$\longrightarrow$ Analytical Euler $G_{\mathrm{I}} / G$

- $44 \times 2$ Numerical Euler $G_{\mathrm{l}} / G$

$\longleftarrow$ Analytical Euler $G_{\mathrm{II}} / G$

- $44 \times 2$ Numerical Euler $G_{\mathrm{II}} / G$

Analytical Timo. $G_{\mathrm{I}} / G$

Analytical Timo. $G_{\mathrm{II}} / G$

$\Delta \quad 880 \times 2$ Numerical Timo. $G_{l} / G$

A $880 \times 2$ Numerical Timo. $G_{\mathrm{II}} / G$

- - Averaged Timo. \& Euler $G_{\mathrm{I}} / G$

- 2D FEM $110 \times 24 G_{l} / G$

$-\cdots$ Averaged Timo. \& Euler $G_{\mathrm{II}} / G$

- 2 D FEM $110 \times 24 G_{\text {III }} / G$

Figure 6: Comparison between various theories for laminated composite DCB energy release rate partitions with varying $N_{2}\left(M_{1}=1, M_{2}=0, N_{1}=0, P_{1}=0, P_{2}=0\right)$. 


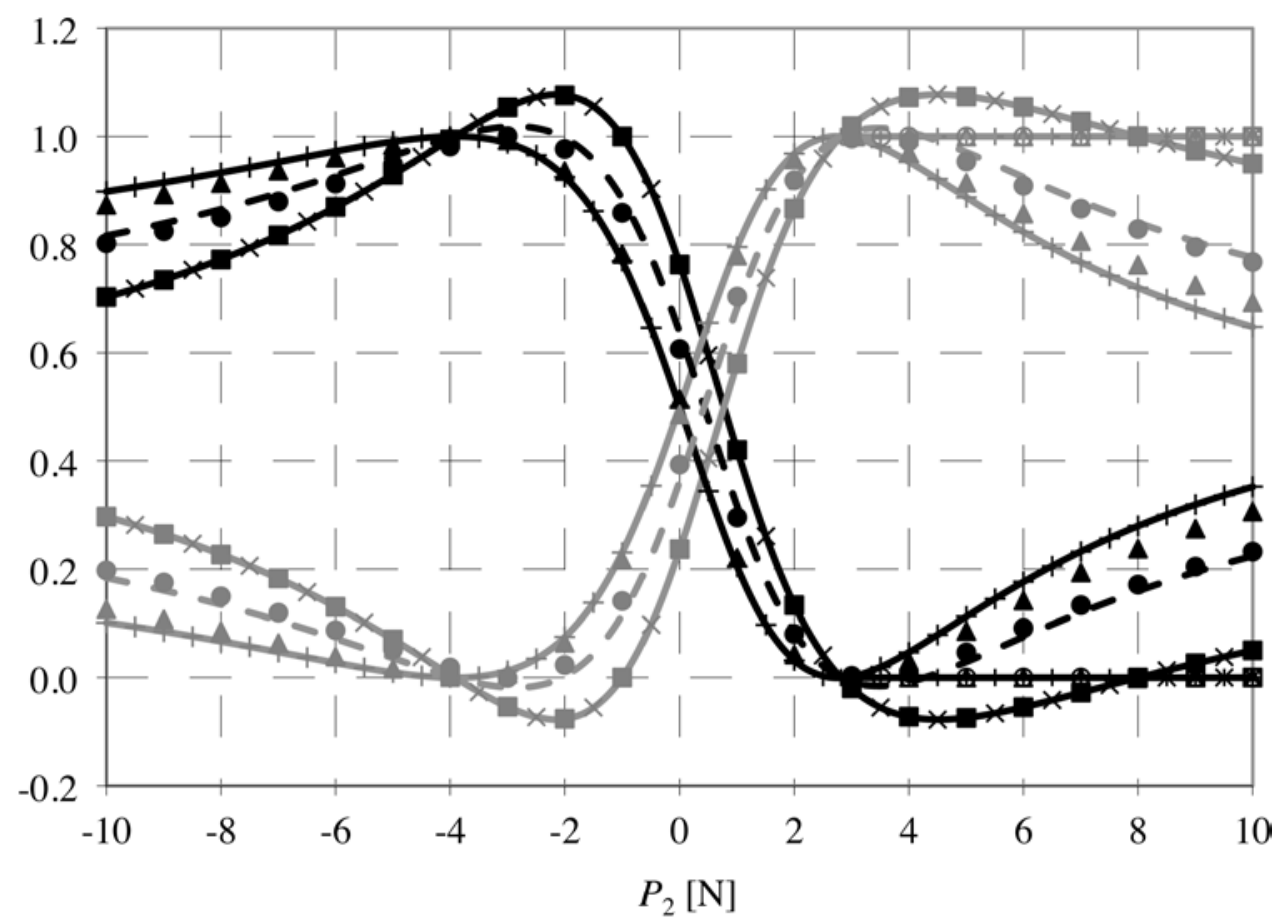

$\longleftarrow$ Analytical Euler $G_{\mathrm{I}} / G$

$\longleftarrow$ Analytical Euler $G_{\mathrm{II}} / G$

- $100 \times 2$ Numerical Euler $G_{l} / G$

- $100 \times 2$ Numerical Euler $G_{\mathrm{II}} / G$

- Analytical Timo. $G_{\mathrm{I}} / G$

- Analytical Timo. $G_{\mathrm{II}} / G$

- $800 \times 2$ Numerical Timo. $G_{\mathrm{I}} / G$

A $800 \times 2$ Numerical Timo. $G_{\mathrm{II}} / G$

- Averaged Timo. \& Euler $G_{\mathrm{I}} / G$

- Averaged Timo. \& Euler $G_{\mathrm{II}} / G$

- 2 D FEM $400 \times 6 G_{\mathrm{l}} / G$ - 2 D FEM $400 \times 6 G_{\text {III }} / G$

Figure 7: Comparison between various theories for clamped-clamped isotropic beam energy release rate partitions with varying $P_{2}$ and $P_{1}=1 \mathrm{~N}$. 


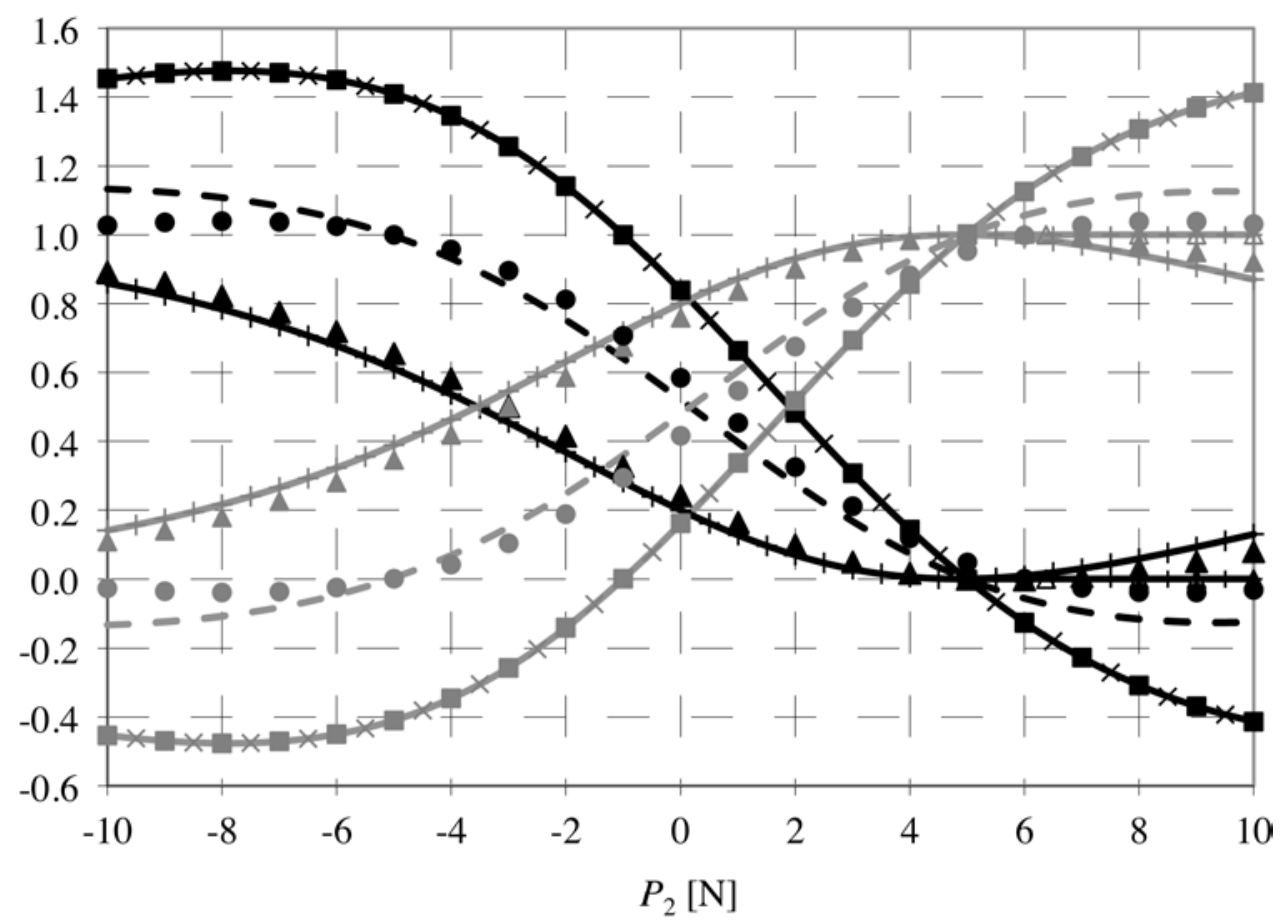

$\longleftarrow$ Analytical Euler $G_{\mathrm{II}} / G$

- $100 \times 2$ Numerical Euler $G_{l} / G$

- $100 \times 2$ Numerical Euler $G_{\mathrm{II}} / G$

- Analytical Timo. $G_{\mathrm{I}} / G$

- Analytical Timo. $G_{\mathrm{II}} / G$

\ $800 \times 2$ Numerical Timo. $G_{\mathrm{I}} / G$

- $800 \times 2$ Numerical Timo. $G_{\mathrm{II}} / G$

- Averaged Timo. \& Euler $G_{\mathrm{I}} / G$

- Averaged Timo. \& Euler $G_{\mathrm{II}} / G$

- 2D FEM $200 \times 16 G_{\mathrm{l}} / G$ - 2 D FEM $200 \times 16 G_{\mathrm{II}} / G$

Figure 8: Comparison between various theories for clamped-clamped composite laminated beam energy release rate partitions with varying $P_{2}$ and $P_{1}=1 \mathrm{~N}$. 
Table 1: Data for numerical simulations of an isotropic DCB.

$\begin{array}{cc}\text { Elastic modulus, } E & 1 \\ \text { Shear modulus, } G_{x} & 1 \\ \text { Poisson's ratio, } \nu & 0.3 \\ \text { Laminate thicknesses, } h_{1} \text { and } h_{2} & 1 \text { and } 2 \\ \text { Intact length of beam, } L & 100 \\ \text { Length of delamination, } a & 10 \\ \text { Width of beam, } b & 1\end{array}$

Table 2: Data for numerical simulations of a laminated composite DCB.

\begin{tabular}{|c|c|}
\hline Ply longitudinal modulus, $E_{11}$ & $139.3 \mathrm{GPa}$ \\
\hline Ply transverse modulus, $E_{22}$ & $9.72 \mathrm{GPa}$ \\
\hline Out-of-plane modulus, $E_{33}$ & $9.72 \mathrm{GPa}$ \\
\hline In-plane shear modulus, $G_{12}$ & $5.58 \mathrm{GPa}$ \\
\hline Out-of-plane shear moduli, $G_{13}$ & $5.58 \mathrm{GPa}$ \\
\hline Out-of-plane shear moduli, $G_{23}$ & $3.45 \mathrm{GPa}$ \\
\hline In-plane Poisson's ratio, $\nu_{12}$ & 0.29 \\
\hline Out-of-plane Poisson's ratio, $\nu_{13}$ & 0.29 \\
\hline Out-of-plane Poisson's ratio, $\nu_{23}$ & 0.4 \\
\hline Ply thickness, $t_{y}$ & $0.125 \mathrm{~mm}$ \\
\hline Sub-laminate lay-up 1 (top) & $(90 / 0)_{1}$ \\
\hline Sub-laminate lay-up 2 (bottom) & $(0 / 90)_{8}$ \\
\hline Laminate thicknesses, $h_{1}$ and $h_{2}$ & $1 \mathrm{~mm}$ and $2 \mathrm{~mm}$ \\
\hline Intact lengths of beam, $L$ & $100 \mathrm{~mm}$ \\
\hline Length of delamination, $a$ & $10 \mathrm{~mm}$ \\
\hline Width of beam, $b$ & $10 \mathrm{~mm}$ \\
\hline
\end{tabular}


Table 3: Comparison between various theories for isotropic DCB energy release rate partitions with varying $P_{1}\left(M_{1}=100, M_{2}=-200, N_{1}=100, N_{2}=0, P_{2}=0\right)$.

\begin{tabular}{ccccccc}
\hline & \multicolumn{5}{c}{ Left crack tip $G_{r} / G[\%]$} \\
\cline { 2 - 7 }$P_{1}[\mathrm{~N}]$ & $\begin{array}{c}\text { Analytical } \\
\text { Euler }\end{array}$ & $\begin{array}{c}\text { Numerical } \\
\text { Euler }(220 \times 2 \\
\text { Timo. beams) }\end{array}$ & $\begin{array}{c}\text { Analytical } \\
\text { Timo. }\end{array}$ & $\begin{array}{c}\text { Numerical } \\
\text { Timo. }(880 \times 2 \\
\text { Timo. beams) }\end{array}$ & $\begin{array}{c}\text { Averaged } \\
\text { Analytical } \\
\text { (Euler \& } \\
\text { Timo.) }\end{array}$ & $\begin{array}{c}\text { 2D FEM } \\
(330 \times 6 \\
\text { QUAD4s) }\end{array}$ \\
\hline-10 & 50.00 & 53.17 & 70.75 & 68.07 & 55.73 & 57.42 \\
-8 & 87.38 & 89.21 & 96.71 & 95.83 & 91.22 & 90.48 \\
-6 & 103.50 & 104.28 & 99.49 & 99.85 & 101.63 & 99.85 \\
-4 & 107.59 & 107.77 & 94.73 & 95.81 & 101.32 & 99.10 \\
-2 & 107.14 & 106.99 & 89.12 & 90.62 & 98.19 & 95.83 \\
0 & 105.26 & 104.94 & 84.21 & 85.98 & 94.74 & 92.38 \\
2 & 103.08 & 102.65 & 80.19 & 82.15 & 91.62 & 89.31 \\
4 & 100.98 & 100.50 & 76.93 & 79.03 & 88.95 & 86.71 \\
6 & 99.07 & 98.56 & 74.27 & 76.46 & 86.69 & 84.52 \\
8 & 97.39 & 96.85 & 72.08 & 74.34 & 84.77 & 82.67 \\
10 & 95.90 & 95.35 & 70.24 & 72.57 & 83.14 & 81.10 \\
\hline
\end{tabular}

Table 4: Comparison between various theories for laminated composite DCB energy release rate partitions with varying $N_{2}\left(M_{1}=1, M_{2}=0, N_{1}=0, P_{1}=0, P_{2}=0\right)$.

\begin{tabular}{|c|c|c|c|c|c|c|}
\hline \multirow[b]{2}{*}{$N,[\mathrm{kN}]$} & \multicolumn{6}{|c|}{ Left crack tip $G_{I} / G[\%]$} \\
\hline & $\begin{array}{l}\text { Analytical } \\
\text { Euler }\end{array}$ & $\begin{array}{c}\text { Numerical } \\
\text { Euler (44×2 } \\
\text { Timo. beams) }\end{array}$ & $\begin{array}{c}\text { Analytical } \\
\text { Timo. }\end{array}$ & $\begin{array}{c}\text { Numerical } \\
\text { Timo. }(880 \times 2 \\
\text { Timo. beams) }\end{array}$ & $\begin{array}{c}\text { Averaged } \\
\text { Analytical } \\
\text { (Euler \& } \\
\text { Timo.) }\end{array}$ & $\begin{array}{c}\text { 2D FEM } \\
(330 \times 6 \\
\text { QUAD4s) }\end{array}$ \\
\hline-25 & -8.86 & -8.79 & 5.06 & 4.58 & -1.90 & -0.09 \\
\hline-20 & -7.08 & -7.03 & 2.16 & 1.84 & -2.46 & -0.38 \\
\hline-15 & -2.15 & -2.14 & 0.13 & 0.05 & -1.01 & 1.28 \\
\hline-10 & 9.69 & 9.65 & 1.63 & 1.92 & 5.66 & 8.03 \\
\hline-5 & 34.91 & 34.80 & 13.32 & 14.07 & 24.11 & 26.39 \\
\hline 0 & 75.08 & 74.93 & 43.59 & 44.68 & 59.34 & 61.33 \\
\hline 5 & 106.08 & 105.95 & 81.33 & 82.19 & 93.70 & 94.00 \\
\hline 10 & 105.22 & 105.19 & 99.04 & 99.26 & 102.13 & 99.38 \\
\hline 15 & 88.47 & 88.52 & 97.80 & 97.47 & 93.14 & 88.56 \\
\hline 20 & 71.80 & 71.89 & 90.46 & 89.81 & 81.13 & 76.09 \\
\hline 25 & 58.97 & 59.09 & 82.89 & 82.06 & 70.93 & 66.01 \\
\hline
\end{tabular}


Table 5: Data for numerical simulations of a clamped-clamped isotropic beam

\begin{tabular}{cc}
\hline Elastic modulus, $E$ & $70 \mathrm{GPa}$ \\
Shear modulus, $G_{t z}$ & $26 \mathrm{GPa}$ \\
Poisson's ratio, $\nu_{f}$ & 0.35 \\
Laminate thicknesses, $h_{1}$ and $h_{2}$ & $1 \mathrm{~mm}$ and $2 \mathrm{~mm}$ \\
Intact lengths of beam, $L_{1}$ and $L_{2}$ & $10 \mathrm{~mm}$ and $25 \mathrm{~mm}$ \\
Length of delamination, $a$ & $65 \mathrm{~mm}$ \\
Width of beam, $b$ & $10 \mathrm{~mm}$ \\
Loading location, $x_{p}$ & $20 \mathrm{~mm}$ \\
Mode $\theta_{p}$ (zero opening displacement) & -3.9169 \\
Mode $\beta_{p}$ (zero normal stress) & 2.8106 \\
Mode $\theta_{P}^{\prime}$ (zero shear displacement) \\
Mode $\beta_{P}^{\prime}$ (zero shear stress)
\end{tabular}

Table 6: Data for numerical simulations of a clamped-clamped composite laminated beam

\begin{tabular}{cc}
\hline Sub-laminate lay-up 1 (top) & $90 /-45 / 0 / 45$ \\
Sub-laminate lay-up 2 (bottom) & $(45 / 0 /-45 / 90)_{2} / 90 /-45 / 0 / 45$ \\
Laminate thicknesses, $h_{1}$ and $h_{2}$ & $0.5 \mathrm{~mm}$ and $1.5 \mathrm{~mm}$ \\
Intact lengths of beam, $L_{1}=L_{2}=L$ & $25 \mathrm{~mm}$ \\
Length of delamination, $a$ & $50 \mathrm{~mm}$ \\
Width of beam, $b$ & $10 \mathrm{~mm}$ \\
Loading location, $x_{p}$ & $25 \mathrm{~mm}$ \\
Mode $\theta_{P^{\prime}}$ (zero opening displacement) & -26.3501 \\
Mode $\beta_{P^{\prime}}$ (zero normal stress) & 4.9836 \\
Mode $\theta_{P}^{\prime}$ (zero shear displacement) & -1 \\
Mode $\beta_{P}^{\prime}$ (zero shear stress) & 66.9002 \\
\hline
\end{tabular}


Table 7: Comparison between various theories for clamped-clamped isotropic beam energy release rate partitions with varying $P_{2}$ and $P_{1}=1 \mathrm{~N}$.

\begin{tabular}{|c|c|c|c|c|c|c|}
\hline \multirow[b]{2}{*}{$P_{2}[\mathrm{~N}]$} & \multicolumn{6}{|c|}{ Left crack tip $G_{I} / G[\%]$} \\
\hline & $\begin{array}{l}\text { Analytical } \\
\text { Euler }\end{array}$ & $\begin{array}{c}\text { Numerical } \\
\text { Euler }(100 \times 2 \\
\text { Timo. beams) }\end{array}$ & $\begin{array}{c}\text { Analytical } \\
\text { Timo. }\end{array}$ & $\begin{array}{l}\text { Numerical } \\
\text { Timo. (800×2 } \\
\text { Timo. beams) }\end{array}$ & $\begin{array}{c}\text { Averaged } \\
\text { Analytical } \\
\text { (Euler \& } \\
\text { Timo.) }\end{array}$ & $\begin{array}{c}\text { 2D FEM } \\
(400 \times 6 \\
\text { QUAD4s) }\end{array}$ \\
\hline-10 & 70.30 & 70.30 & 89.83 & 87.39 & 81.64 & 80.23 \\
\hline-8 & 77.28 & 77.27 & 93.31 & 91.38 & 86.53 & 85.05 \\
\hline-6 & 86.94 & 86.93 & 97.26 & 96.09 & 92.83 & 91.28 \\
\hline-4 & 99.46 & 99.45 & 99.99 & 99.92 & 99.76 & 98.13 \\
\hline-2 & 107.63 & 107.62 & 92.53 & 93.60 & 99.70 & 97.69 \\
\hline 0 & 76.34 & 76.34 & 49.91 & 51.43 & 63.66 & 60.69 \\
\hline 2 & 13.35 & 13.36 & 3.19 & 4.24 & 8.26 & 8.07 \\
\hline 4 & -7.25 & -7.23 & 4.70 & 3.05 & -0.78 & 0.91 \\
\hline 6 & -5.45 & -5.44 & 17.66 & 14.24 & 7.53 & 9.10 \\
\hline 8 & 0.00 & 0.01 & 27.98 & 23.76 & 15.98 & 17.14 \\
\hline 10 & 5.01 & 5.02 & 35.22 & 30.65 & 22.42 & 23.25 \\
\hline
\end{tabular}

Table 8: Comparison between various theories for clamped-clamped composite laminated beam energy release rate partitions with varying $P_{2}$ and $P_{1}=1 \mathrm{~N}$.

\begin{tabular}{|c|c|c|c|c|c|c|}
\hline \multirow[b]{2}{*}{$P_{2}[\mathrm{~N}]$} & \multicolumn{6}{|c|}{ Left crack tip $G_{I} / G[\%]$} \\
\hline & $\begin{array}{l}\text { Analytical } \\
\text { Euler }\end{array}$ & $\begin{array}{c}\text { Numerical } \\
\text { Euler }(100 \times 2 \\
\text { Timo. beams) }\end{array}$ & $\begin{array}{c}\text { Analytical } \\
\text { Timo. }\end{array}$ & $\begin{array}{l}\text { Numerical } \\
\text { Timo. }(800 \times 2 \\
\text { Timo. beams) }\end{array}$ & $\begin{array}{c}\text { Averaged } \\
\text { Analytical } \\
\text { (Euler \& } \\
\text { Timo.) }\end{array}$ & $\begin{array}{l}\text { 2D FEM } \\
\text { (200×16 } \\
\text { QUAD4s) }\end{array}$ \\
\hline-10 & 145.33 & 145.33 & 85.89 & 89.20 & 113.28 & 101.99 \\
\hline-8 & 147.63 & 147.62 & 78.35 & 82.13 & 110.80 & 100.40 \\
\hline-6 & 145.01 & 145.00 & 67.71 & 71.93 & 104.63 & 95.65 \\
\hline-4 & 134.61 & 134.60 & 53.62 & 58.15 & 93.11 & 86.17 \\
\hline-2 & 114.10 & 114.09 & 36.84 & 41.43 & 75.21 & 70.87 \\
\hline 0 & 83.82 & 83.81 & 19.96 & 24.13 & 52.03 & 50.73 \\
\hline 2 & 48.24 & 48.24 & 6.91 & 9.90 & 27.66 & 29.71 \\
\hline 4 & 14.30 & 14.30 & 0.57 & 1.65 & 7.40 & 12.78 \\
\hline 6 & -12.60 & -12.61 & 0.96 & -0.24 & -5.64 & 2.50 \\
\hline 8 & -30.77 & -30.77 & 5.90 & 2.59 & -11.60 & -1.66 \\
\hline 10 & -41.30 & -41.29 & 12.98 & 7.99 & -12.46 & -1.69 \\
\hline
\end{tabular}


Table 9: Data of a clamped circular plate with a central fracture.

\begin{tabular}{|c|c|c|c|c|c|c|c|c|}
\hline$E$ & $\nu$ & $R$ & $a$ & $h_{1}$ & $h_{2}$ & $\gamma$ & $\beta_{p}$ & $\theta_{r}$ \\
\hline $140 \mathrm{GPa}$ & 0.3 & $100 \mathrm{~mm}$ & $20 \mathrm{~mm}$ & $1 \mathrm{~mm}$ & $2 \mathrm{~mm}$ & 2 & $20 / 7$ & -4 \\
\hline
\end{tabular}

Table 10: Energy release rates $\left(\mu \mathrm{J} / \mathrm{m}^{2}\right)$ for $\theta_{P}$ and $\beta_{P}$ modes of the circular plate with a central crack.

\begin{tabular}{|c|c|c|c|c|c|c|c|c|c|}
\hline & \multicolumn{3}{|c|}{40 elements } & \multicolumn{3}{|c|}{200 elements } & \multicolumn{3}{|c|}{ Analytical } \\
\hline & $G_{I}$ & $G_{H}$ & $G$ & $G_{I}$ & $G_{H}$ & $G$ & $G_{1}$ & $G_{H H}$ & $G$ \\
\hline$\theta_{\mu}$ & 740.9 & 0 & 740.9 & 675.2 & 0 & 675.2 & 658.6 & 0 & 658.6 \\
\hline$\beta_{p}$ & 0 & 408.3 & 408.3 & 0 & 372.0 & 372.0 & 0 & 362.9 & 362.9 \\
\hline
\end{tabular}

